\title{
Investigation of Electron Heat Pinch in ASDEX-Upgrade
}

\author{
P. Mantica ${ }^{1}$, F. Ryter ${ }^{2}$, C. Capuano ${ }^{3}$,H.U. Fahrbach ${ }^{2}$, F. Leuterer ${ }^{2}$, W. Suttrop ${ }^{2}$, \\ J. Weiland ${ }^{4}$ and ASDEX-Upgrade Team
}

\author{
${ }^{1}$ Istituto di Fisica del Plasma, EURATOM-ENEA-CNR Association, Milan, Italy \\ ${ }^{2}$ Max-Planck-Institut für Plasmaphysik, EURATOM Association, Garching, Germany \\ ${ }^{3}$ Dept. of Physics, University of Milano-Bicocca, Milano, Italy - present address: ITER \\ EU team, Garching, Germany \\ ${ }^{4}$ Chalmers University of Technology and Euratom-VR Association, Göteborg Sweden
}

\begin{abstract}
The existence of an electron heat pinch has been experimentally investigated in ASDEXUpgrade in plasmas with strong off-axis Electron Cyclotron Heating (ECH). Localization of $1.6 \mathrm{MW}$ of ECH power at $\rho=0.65$ in plasmas with low Ohmic power $(<100 \mathrm{~kW})$ still resulted in peaked electron temperature $\left(T_{\mathrm{e}}\right)$ profiles at densities $n_{\mathrm{e} 0} \sim 2.710^{19} \mathrm{~m}^{-3} .2$ out of 4 gyrotrons were modulated in order to study convective effects also on the $T_{\mathrm{e}}$ transient response at different frequencies. Peculiar profiles of the modulation amplitude have been measured, very flat in the region just inside $\rho \quad(0.3<\rho<0.65)$ or even increasing rather than decreasing away from $\rho$ on the inner side. The phase profiles are instead regularly indicating the propagation of the heat wave in both directions away from $\rho$. Power balance analysis shows that the heat flux in the core region is very close to zero, but uncertainties do not allow a clear determination of its sign. Modelling steady-state and modulation results with an empirical model featuring a diffusive term characterized by a critical gradient length $\left(\mathrm{L}_{\mathrm{Te}}=-\mathrm{T}_{\mathrm{e}} / \nabla \mathrm{T}_{\mathrm{e}}\right)$ behaviour and a constant Gaussian shaped heat pinch profile allows good reproduction of the data when the region just inside $\rho$ is close to the critical threshold and oscillating around it, and a small convective term $U \sim 1-2 \mathrm{~m} / \mathrm{s}$ is present in the same region. At higher density the $T_{e}$ profile becomes flatter and the amplitude profiles recover the usual shape with stronger decay inside with respect to outside $\rho$. In this case no heat pinch is needed and the plasma goes below the critical threshold inside $\rho$. The results are consistent with the theoretical prediction of a turbulence regulated by a threshold in $\mathrm{R} / \mathrm{L}_{\mathrm{Te}}$ and a small turbulence generated electron heat pinch term. However initial attempts to perform first principle based simulations using the quasi-linear electrostatic drift wave Weiland model have failed to reproduce the experimental results.
\end{abstract}

\section{Introduction}

A number of experiments (DIII-D[1], RTP[2], FTU[3]) have reported that, in conditions of localised off-axis electron heating, inward electron heat convection (i.e. a term in the heat flux proportional to the temperature $\left(T_{\mathrm{e}}\right)$ rather than to its gradient) is observed. Experimentally, the heat pinch can be identified by power balance analysis in steady-state plasmas only if negative values of the electron heat diffusivity are obtained, as reported in $[1,3]$. A more suitable tool is modulated Electron Cyclotron Heating $(\mathrm{ECH})$, since in this case the inward convection may also be identified from distortions of the profile of the heat wave amplitude at low frequency, as reported in [2].

From a theoretical perspective, a deeper understanding of turbulent mechanisms that lead to the onset of particle and heat pinches has been gained [4-8]. The concept of the existence of a critical value of the inverse temperature gradient length $\left(\mathrm{R} / \mathrm{L}_{\mathrm{Te}}=-\mathrm{R} \nabla \mathrm{T}_{\mathrm{e}} / \mathrm{T}_{\mathrm{e}}\right)$ above which turbulence sets in and determines particle and heat transport, as predicted by 
the theory of electrostatic Ion Temperature Gradient/ Trapped Electron Modes (ITG/TEM) instabilities, is now supported experimentally by many recent results in the field of electron transport [9-15]. This theory predicts also the existence of turbulence driven convective heat fluxes either due to the off-diagonal contribution of the $\nabla n_{e}$ term or driven by the magnetic field in homogeneity $(\nabla B)[4,7,8]$. Also convective terms arising from the interaction of turbulence with MHD modes have been predicted in the framework of non-linear electromagnetic fluid turbulence as discussed in [8].

All these experimental and theoretical results justify further experimental attempts to investigate heat pinches. Since in the presence of central heating, i.e. large heat flux, diffusive transport is always dominant, making it difficult to recognize the presence of possible convective terms, off-axis heating needs to be used. The difficulty of such experiments is that the need to suppress central sources to recognize the existence of pinches from power balance analysis leads inevitably to disappearance of core turbulence and therefore of both turbulent diffusive transport and of the mechanisms providing the pinch itself. In this sense heat pinch experiments differ from analogous experiments for particle pinches, in which the central particle source can be suppressed without suppressing core turbulence, which is maintained by the central heat source. It is worth mentioning that a similar difficulty, as will be discussed later, characterizes also all turbulent simulations addressing situations of strongly off-axis heated plasmas, in which the central source driving the turbulence becomes small. For heat pinch experiments and simulations it becomes therefore even more important to support steady-state profile analysis with transient transport results from power modulation.

A dedicated set of modulated ECH experiments has been carried out on ASDEXUpgrade to investigate the presence of heat pinch components in the electron heat flux in conditions of strong off-axis ECH power $\left(\mathrm{P}_{\mathrm{ECH}} / \mathrm{P}_{\mathrm{OH}}>10\right)$. The experiment relies on the capabilities on AUG to achieve far off-axis localization of a large amount of ECH power and to measure the $T_{\mathrm{e}}$ response to modulation with a well space- and time- resolved ECE diagnostic. This paper describes the experimental results, their transport analysis and modelling using semi-empirical models for the electron heat diffusivity $\left(\chi_{e}\right)$, and an attempt to model the results using the first principle quasi-linear electrostatic drift-wave based Weiland model [4].

\section{Experimental set-up}

The ECH system at ASDEX Upgrade consists of 4 gyrotrons providing 1.6 MW at 140 $\mathrm{GHz}$ which corresponds to the $2^{\text {nd }}$ harmonic of the electron cyclotron frequency. The power is injected in the $\mathrm{X}$-mode from the low field side without toroidal incidence. The deposition radius $(\rho \quad)$ was varied between $\rho_{\text {tor }}=0.2$ and 0.65 using either a finite angle for the launcher mirror to steer each beam poloidally at fixed $\mathrm{B}_{\mathrm{T}}$ or by setting this angle to about zero to obtain deposition in the equatorial plane and adjusting the $\mathrm{B}_{\mathrm{T}}$ value only to achieve the required off-axis location. We consider the latter scheme safer for these studies since it allows to exclude the possibility of spurious central ECH power. In fact with the resonance on the high field side there is no $\mathrm{ECH}$ resonance (cold or warm) inside the deposition radius, so no possibility of spurious core absorption. The experiments presented in the following have all been performed with deposition in the equatorial plane. The power was modulated with a square waveform between 1.6 and $0.8 \mathrm{MW}$ with $f=14.7 \mathrm{~Hz}$ or $f=29.4 \mathrm{~Hz}$ and duty-cycle $d_{\mathrm{c}}=0.88$. The power of each ECRH beam is measured at the torus entrance by calibrated detectors with a sampling frequency of $1 \mathrm{MHz}$. These experimentally determined power levels have then been employed as a known input in all simu- 
lations described in the paper. Some cases with a smaller square waveform amplitude between 1.6 and $1.45 \mathrm{MW}$ and with $d_{\mathrm{c}}=0.5$ have been performed to check the dependence of results on the amplitude of the power modulation. Plasma discharges with $B_{\mathrm{T}}=2-2.5 \mathrm{~T}$, $I_{\mathrm{p}}=400 \mathrm{kA}, \bar{n}_{e}=2-3.510^{19} \mathrm{~m}^{-3}$ have been used as targets. Even with the outermost ECH deposition the $1^{\text {st }}$ pass ECH absorption calculated with a beam tracing code is always larger than $99 \%$.

The $T_{\mathrm{e}}$ profile evolution was measured by a 60 channel ECE heterodyne radiometer, with a space resolution for each channel of about $1 \mathrm{~cm}$ and a distance between channels of 2-3 cm. The ECE diagnostic is calibrated absolutely yielding for most of the channels an experimental uncertainty of about $7 \%$. In addition, for the present study a plasma discharge with a $B_{T}$ ramp was performed allowing a relative re-calibration of a few obvious outliers. An indication of the ion temperature value in the centre was obtained by neutral particle analyzers (NPA). At such low densities the $T_{i}$ values yielded by the analysis are rather accurate because the attenuation of the neutral flux from the center is weak. The experimental uncertainty on $\mathrm{T}_{\mathrm{i} 0}$ is about $20 \%$. Density profile measurements were made by Thomson Scattering and interferometer, with an uncertainty of the order of $15 \%$.

Standard Fast Fourier Transform techniques have been applied during the stationary phase of the discharge to obtain the spatial profiles of amplitude (A) and phase $(\varphi)$ of the $\mathrm{T}_{\mathrm{e}}$ perturbation at different harmonics of the modulation frequency $\omega$. Phases are always calculated with respect to the ECH power waveform. As described in [16] the slopes of A and $\varphi$ profiles yield an estimate of the incremental heat diffusivity $\chi_{e}{ }^{h p}=-1 / n_{e} \partial q_{e} / \partial \nabla T_{e}$ given by:

$$
\chi_{e}^{h p}=\frac{\frac{3}{4} \omega}{-\varphi^{\prime}\left(\frac{A^{\prime}}{A}+\frac{1}{2 r}-\frac{1}{2 r_{n}}\right)} \quad \text { Eq. (1) }
$$

where $r_{n}=-n_{e} / \nabla n_{e}$. Therefore flat $A$ and $\varphi$ profiles correspond to a situation of high $\chi_{e}{ }^{\text {hp }}$, while low values of $\chi_{\mathrm{e}}^{\mathrm{hp}}$ yield very peaked profiles of $\mathrm{A}$ and $\varphi$. The slopes of $\mathrm{A}$ and $\varphi$ separately yield two values

$\chi^{A}=\frac{\frac{3}{4} \omega}{\left(\frac{A^{\prime}}{A}+\frac{1}{2 r}-\frac{1}{2 r_{n}}\right)^{2}} \quad$ and $\quad \chi^{\varphi}=\frac{\frac{3}{4} \omega}{\varphi^{\prime 2}} \quad \operatorname{Eq}(2)$

which will be used in the following to quantify the behaviour of A and $\varphi$ profiles. In simple diffusive cases $\chi^{\mathrm{A}} \leq \chi^{\varphi}$, the difference being due to damping terms (i.e. source terms in the linearized heat transport equation proportional to the temperature fluctuation) whose effect vanishes at high frequency [16].

\section{Experimental results}

\subsection{Low density discharges}

Fig. 1 shows the time evolution of the electron temperature for a discharge $\left(B_{\mathrm{T}}=2.05 \mathrm{~T}\right.$, $\left.I_{\mathrm{p}}=400 \mathrm{kA}, \bar{n}_{e}=210^{19} \mathrm{~m}^{-3}, \mathrm{q}_{\text {edge }} \sim 8\right)$ where ECH deposition was located at $\rho_{\text {tor }}=0.65$ with deposition on the equatorial plane. Discharges with smaller values of $\rho_{\text {dep }}$ show very similar phenomenology, so in this paper we restrict to cases with $\rho_{\text {dep }}=0.65$ where results are most outstanding and the analysis is made easier by the larger width of the region inside the ECH deposition. ECH modulation at $14.7 \mathrm{~Hz}$ with amplitude $50 \%$ and $d_{\mathrm{c}}=0.88$ was applied in the time interval $\mathrm{t}=2-4 \mathrm{~s}$. Sawteeth are suppressed after $\mathrm{t}=2.5 \mathrm{~s}$. The $T_{\mathrm{e}}$ evolution in the interval $t=2-3 \mathrm{~s}$ in Fig. 1 is dominated by the ECH switch-on and the current broadening following off-axis heating. However it can be immediately noticed from Fig. 1 that the increase in central $T_{\mathrm{e}}$ is comparable in magnitude to the increase at $\rho_{\mathrm{dep}}$. Fig.2, showing the initial evolution of $\mathrm{T}_{\mathrm{e}}$ profiles after ECH switch-on, indicates that such increase takes 
place on a slightly slower time scale than the one at $\rho_{\text {dep }}$, but is still quite fast. Suprathermal contributions can be excluded as the ECH resonance is placed on the high field side. This core response is not compatible with simple diffusive arguments unless a very high value of $\chi_{e}$ is present in the core plasma. In fact, the increase in central $T_{e}$ occurs although the only heating in this region is provided by the Ohmic power which is small and decreases after the ECH switch-on, due to the temperature increase and to the current diffusion following the broadening of the $\mathrm{T}_{\mathrm{e}}$ profile. In a conventional diffusive situation with $\chi_{e}$ increasing from low in the core to high in the edge, one would expect a much smaller and slower $\mathrm{T}_{\mathrm{e}}$ increase in the centre with respect to the one at ECH deposition. To investigate the transport properties of the discharge however we will not use this large initial transient, but we shall mainly concentrate on the quasi-stationary phase between $t=3.2 \mathrm{~s}$ and $\mathrm{t}=4 \mathrm{~s}$, where a much smaller $\mathrm{T}_{\mathrm{e}}$ perturbation takes place on top of a stationary situation. Fig. 3 shows the changes of the $T_{\mathrm{e}}$ profile during one cycle of modulation in this phase. It shows that the $T_{e}$ modulation is small. One also notices little reduction in amplitude towards the edge compared to that towards the centre, which reflects the low transport in the core region. We will come back with some considerations on the switch-on phase in Sect.4.4.

Figure 1. Experimental time traces of ECE $T_{e}$ at various radii inside ECH deposition for AUG shot 17175 . ECH is deposited at $\rho_{\text {tor }}=0.65$.

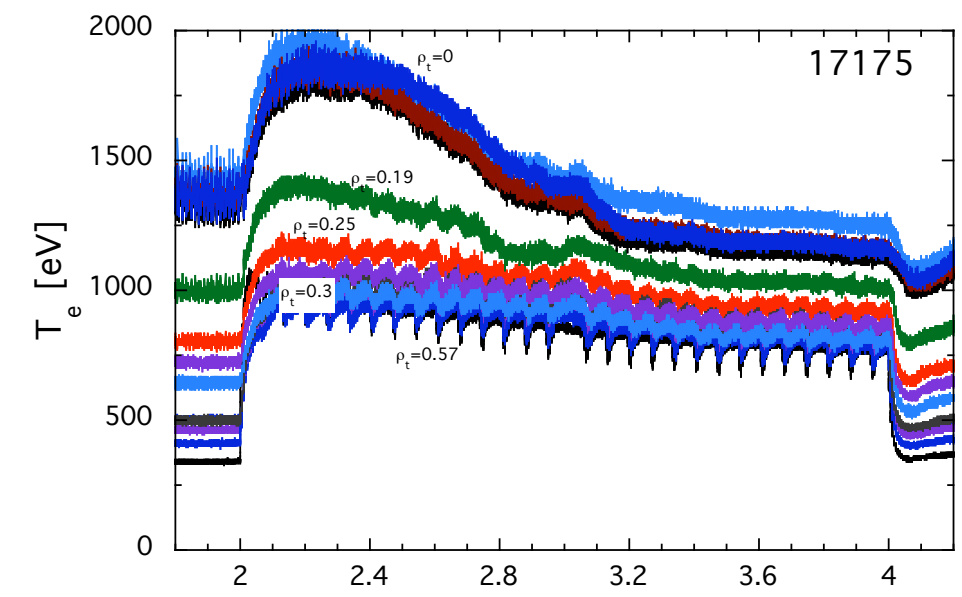

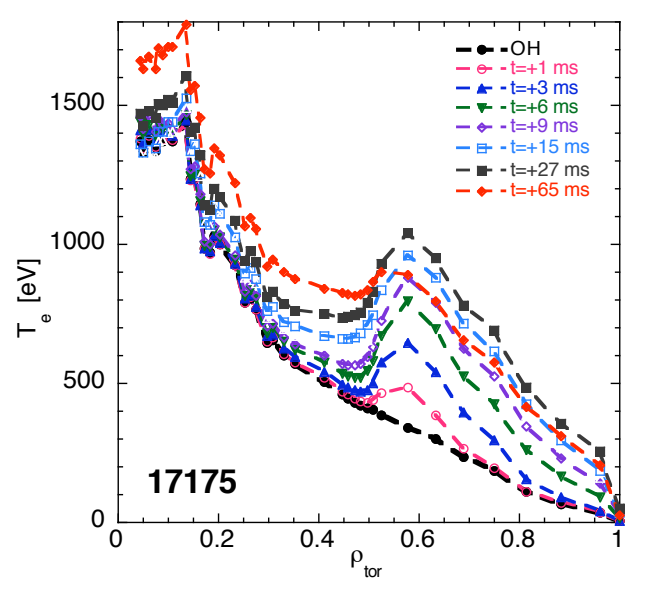

Figure 2. Time evolution of $T_{e}$ profiles at $\mathrm{ECH}$ switch-on from $\mathrm{OH}$ phase for shot 17175. Time refers to the ECH start time.

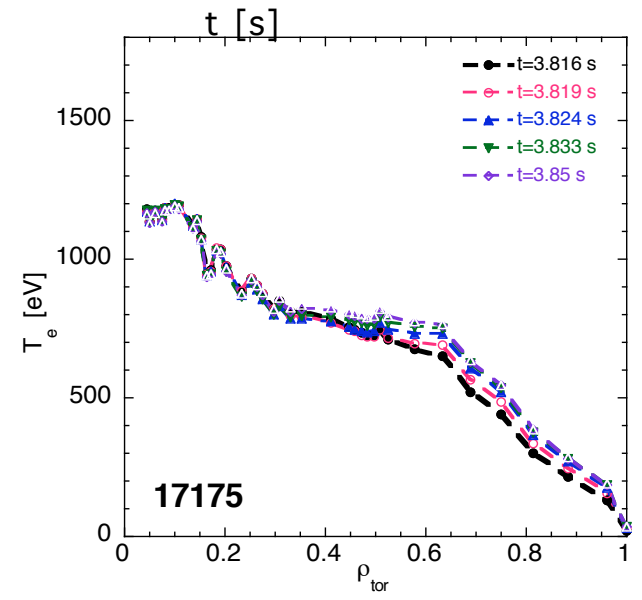

Figure 3. Time evolution of $\mathrm{T}_{\mathrm{e}}$ profiles during one MECH cycle in the stationary phase.

Fig. 4 shows the measured time-averaged profiles of $T_{\mathrm{e}}$ and $n_{\mathrm{e}}$ in the quasi stationary phase $\mathrm{t}=3.2-4 \mathrm{~s} . T_{\mathrm{i} 0}$ from NPA is $\sim 600 \mathrm{eV}$ in Ohmic phase and $\sim 900 \mathrm{eV}$ during ECH. One can see that the $T_{\mathrm{e}}$ profile remains peaked in the centre in spite of the large power applied 
off-axis. Figs.5a-b show the profiles of amplitude and phase of the $T_{\mathrm{e}}$ wave at the first 5 harmonics of the modulation frequency. For each harmonic only experimental points for which peaks above noise level are observed in the spectra are plotted. The noise level is estimated for each harmonic by averaging the signal level on each side of the thin peak corresponding to the considered frequency. The rather low modulation frequency of 14.7 $\mathrm{Hz}$ was chosen to maximize the sensitivity of the amplitude profile to heat pinch terms. One can notice that, at the lowest harmonics, amplitudes in the region $0.3<\rho<0.65$ are very flat, at $1^{\text {st }}$ harmonic even flatter than in the region outside $\rho_{\text {dep }}$. This is not the case for phase profiles, which always show as expected a higher slope inside with respect to outside $\rho_{\text {dep }}$ (although we note that the external part of the $\varphi$ and, to less extent, A profiles at the lowest frequencies is not only determined by transport but heavily influenced by the presence of the plasma edge [16]). Inside $\rho=0.3$ instead there is a change of slope and the amplitudes decay more strongly. Such a change is also visible on the phase profiles although less clearly. The amplitude profile in the region $0.3<\rho<0.65$ is also much flatter than the phase profile: this can be quantified in terms of comparison of $\chi^{\mathrm{A}}$ and $\chi^{\varphi}$ defined in Sect.2 (Eq.(2)). Considering the $1^{\text {st }}$ harmonic, we obtain for the region $0.4<\rho_{\text {tor }}<0.65 \chi^{\mathrm{A}}=4.6 \mathrm{~m}^{2} / \mathrm{s}$ and $\chi^{\varphi}=2.2 \mathrm{~m}^{2} / \mathrm{s}$, whilst with a simple diffusive model one should have $\chi^{\mathrm{A}} \leq \chi^{\varphi}$. This discrepancy between $\chi^{\mathrm{A}}$ and $\chi^{\varphi}$ reduces for increasing frequency until at $4^{\text {th }}$ harmonic we get $\chi^{\mathrm{A}}=\chi^{\varphi}=2.8 \mathrm{~m}^{2} / \mathrm{s}$. For the region $0<\rho_{\text {tor }}<0.3$ instead we recover a diffusive behaviour with $\chi^{\mathrm{A}}=\chi^{\varphi}=\chi^{\mathrm{hp}}=0.22 \mathrm{~m}^{2} / \mathrm{s}$.

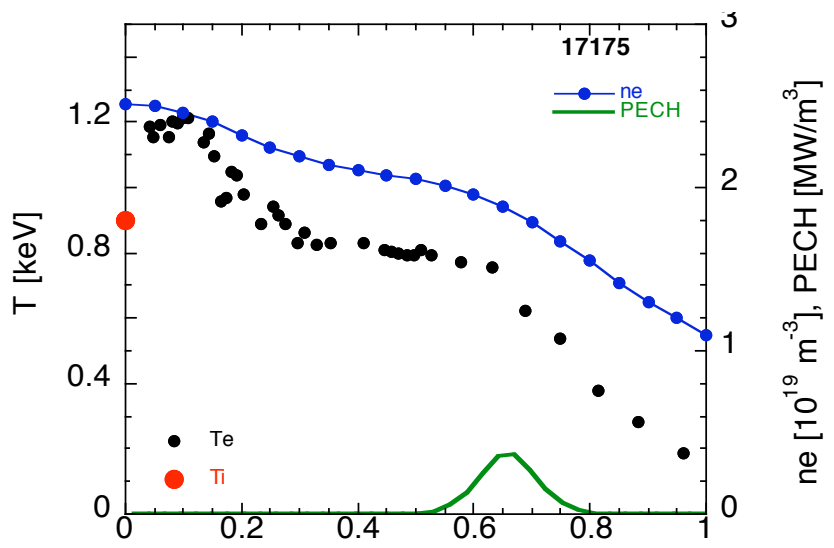

Figure 4. Time-averaged profiles of $\mathrm{T}_{\mathrm{e}}$ and $\mathrm{n}_{\mathrm{e}}$ during the stationary phase $\mathrm{t}=3.2-4 \mathrm{~s}$ for shot 17175 (low density, high modulation amplitude). The ECH power density at power $\mathrm{ON}$ is also plotted, as well as the value of $\mathrm{T}_{\mathrm{i} 0}$.
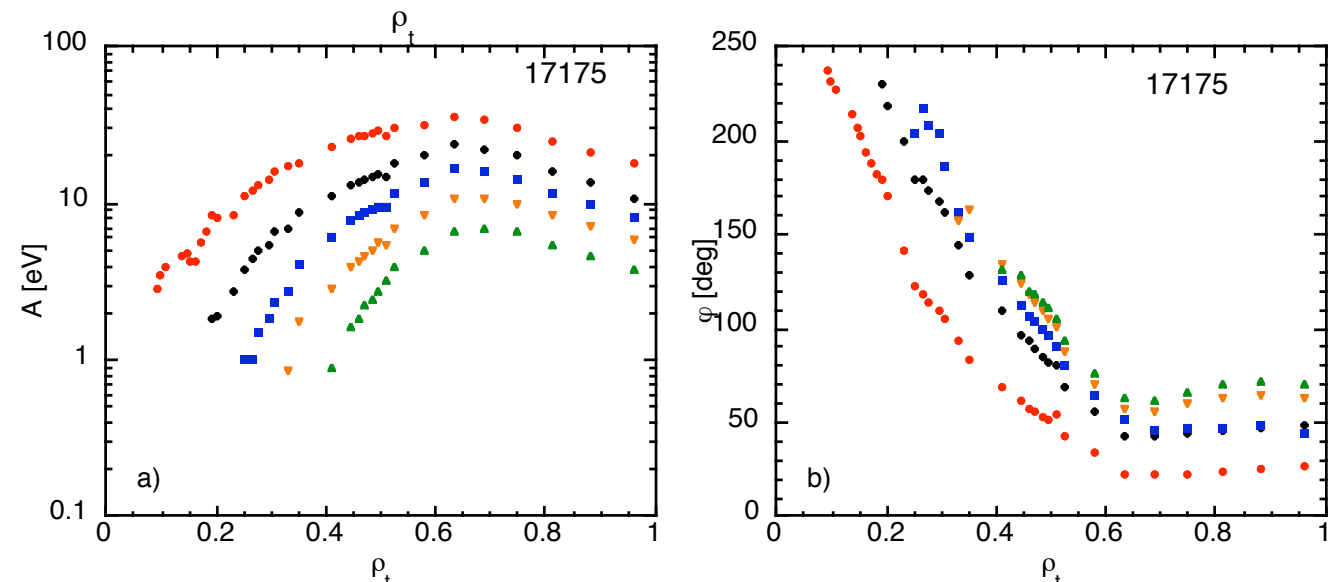

Figure 5. Profiles of amplitude (a) and phase relative to ECH power (b) of the $\mathrm{T}_{\mathrm{e}}$ heat wave at 5 harmonics of the modulation frequency for discharge 17175. $t=3.2-4$ s. From highest to lowest amplitudes: $1^{\text {st }}$ to $5^{\text {th }}$ harmonic. 
Fig.6 and 7a-b show the same kind of results of a shot identical to the previous one but with small modulation amplitude $\left(\Delta_{\mathrm{PECH}}=150 \mathrm{~kW}, \mathrm{~d}_{\mathrm{c}}=0.5\right)$. Due to the $\mathrm{d}_{\mathrm{c}}$ value, all even harmonics are absent from the spectrum.

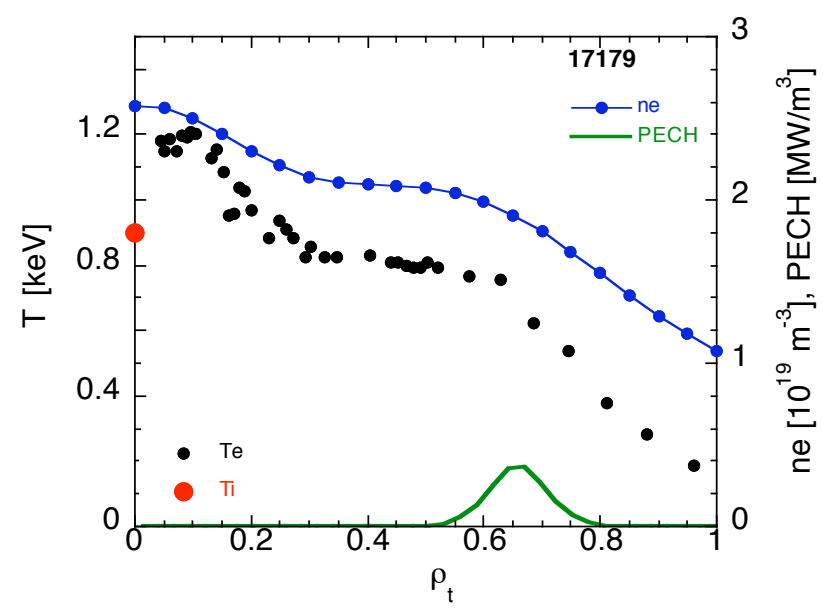

Figure 6. Time-averaged profiles of $T_{e}$, $\mathrm{T}_{\mathrm{i}}, \mathrm{n}_{\mathrm{e}}$ during the stationary phase $\mathrm{t}=3.6-4$ $\mathrm{s}$ for shot 17179 (low density, small modulation amplitude). The ECH power density at power $\mathrm{ON}$ is also plotted.
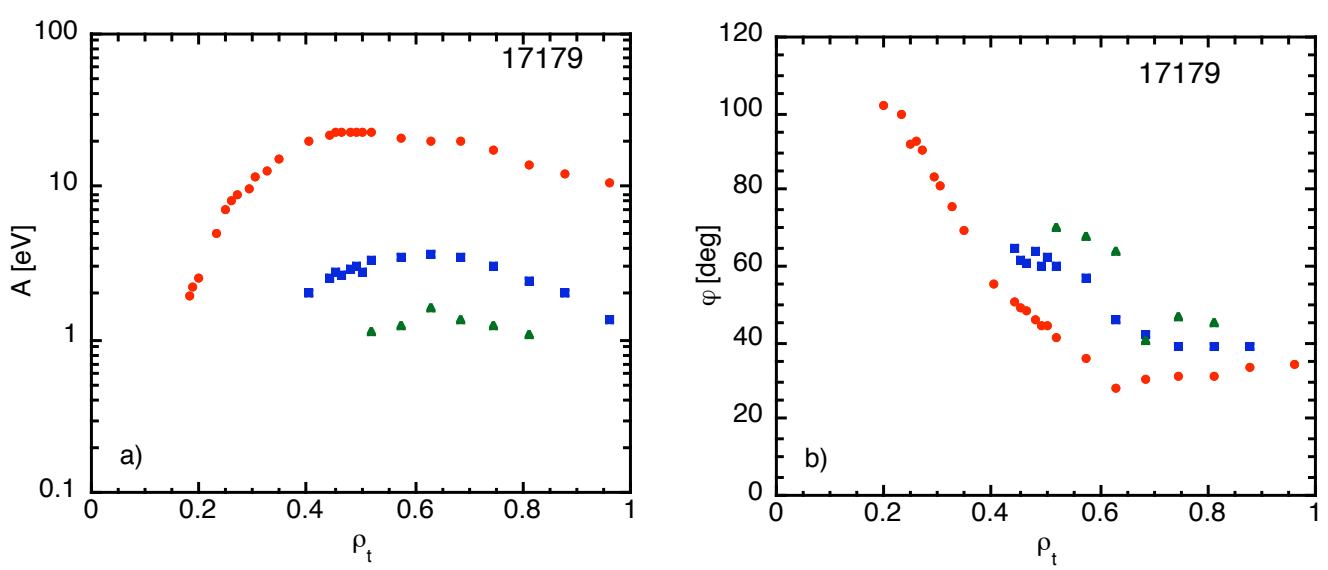

Figure 7. Profiles of amplitude (a) and phase relative to $E C H$ power (b) of the $T_{e}$ heat wave at $1^{\text {st }}, 3^{\text {rd }}, 5^{\text {th }}$ harmonics of the modulation frequency for discharge $17179 . t=3.6-4 \mathrm{~s}$. From highest to lowest amplitudes: $1^{\text {st }}$ to $5^{\text {th }}$ harmonic.

It can be seen that similar results are obtained with small modulation amplitude, with even stronger distortion of the amplitude at $1^{\text {st }}$ harmonic and remaining visible also at $3^{\text {rd }}$ harmonic. This is a confirmation that the amplitude distortion seen in 17175 is not due to the large amplitude of the power modulation. The new important element from this shot is that $1^{\text {st }}$ harmonic amplitudes are even growing instead of decaying in the region $0.4<\rho_{\text {tor }}<0.65$, so that the maximum A lies well inside the deposition radius, whilst the minimum $\varphi$ remains at $\rho_{\text {dep }}$. The A maximum is at $\rho_{\text {dep }}$ only at $3^{\text {rd }}$ and $5^{\text {th }}$ harmonic. This behaviour can only arise in the presence of a convective-like component in the perturbed transport equation. In such region in fact the perturbative diffusivity $\chi^{\text {hp }}$ from Eq.(1) would result of negative sign. These observations remind of the results reported for the RTP tokamak in [2]. The change of amplitude slope at $\rho_{\text {tor }} \sim 0.4$ is also visible as in shot 17175 , and clearly visible also on the phase profiles.

\subsection{High density discharges}

When the line density is progressively increased from $\bar{n}_{e}=210^{19} \mathrm{~m}^{-3}$ to $\bar{n}_{e}=3.310^{19} \mathrm{~m}^{-3}$ (all other parameters being kept the same), the $\mathrm{T}_{\mathrm{e}}$ core value decreases and a hump becomes 
visible at $\rho_{\text {dep }}$, so that a region of positive $\mathrm{T}_{\mathrm{e}}$ gradient appears just inside $\rho_{\text {dep }}$. Figs. 8 and 9a-b show the profiles for the case at $\bar{n}_{e}=2.810^{19} \mathrm{~m}^{-3}$. The amplitude distortion disappears from the modulation data. The amplitude profiles recover the usual shape with stronger decay inside with respect to outside $\rho$, and a slope consistent with the slope of the phase profile. In this shot, consistently with a diffusive behaviour, $\chi^{\mathrm{A}}=\chi^{\varphi}=\chi^{\mathrm{hp}}=0.51$ $\mathrm{m}^{2} / \mathrm{s}$ in the region $0.4<\rho_{\text {tor }}<0.6$.

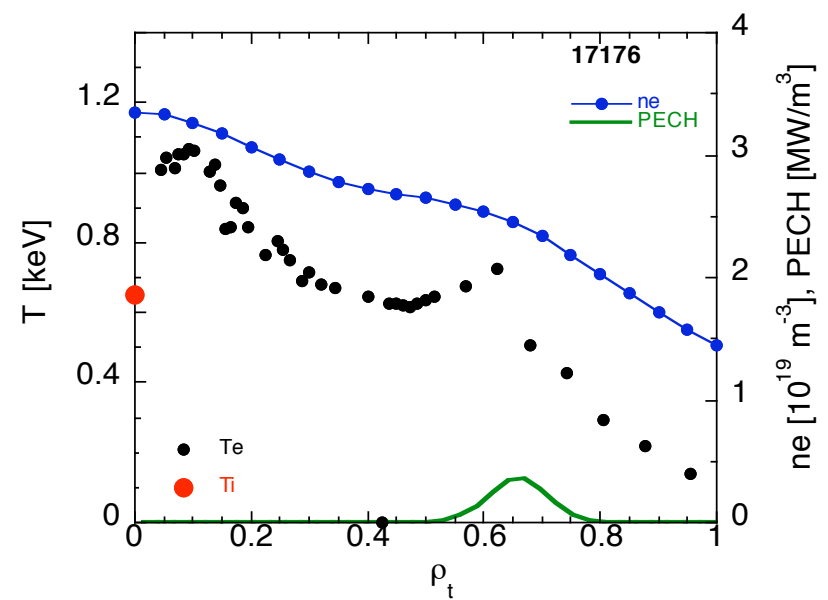

Figure 8. Time-averaged profiles of $T_{e}$, $\mathrm{T}_{\mathrm{i}}, \mathrm{n}_{\mathrm{e}}$ during the stationary phase $\mathrm{t}=3.6-4 \mathrm{~s}$ for shot 17176 (high density). The ECH power density at power $\mathrm{ON}$ is also plotted.
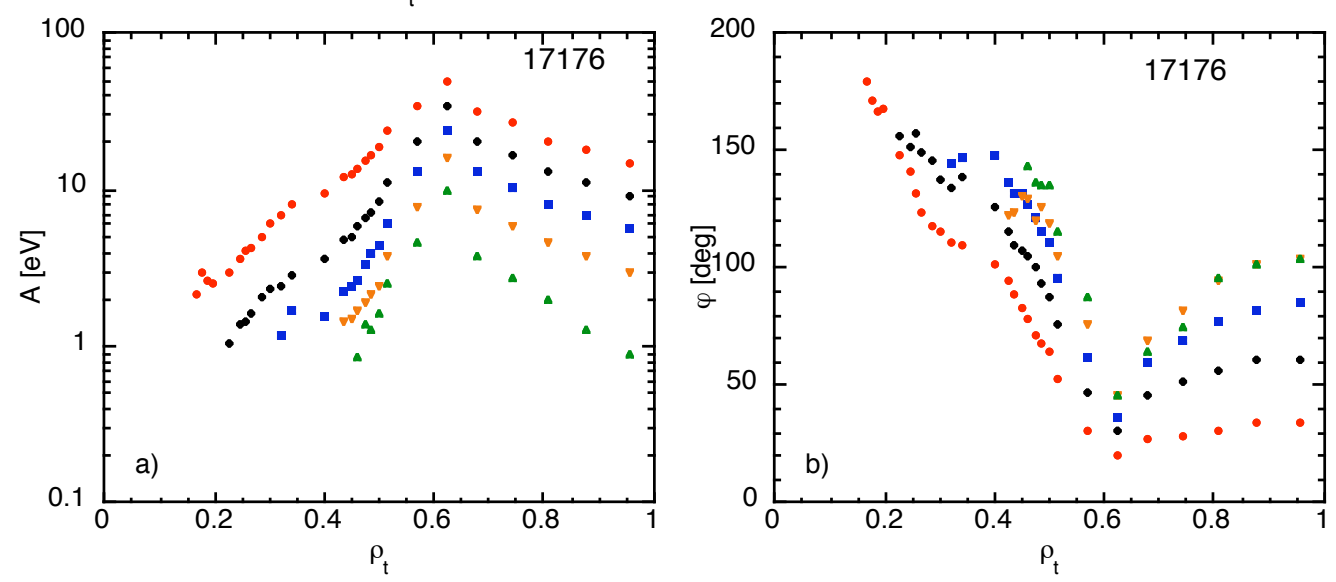

Figure 9. Profiles of amplitude (a) and phase relative to $\mathrm{ECH}$ power (b) of the $\mathrm{T}_{\mathrm{e}}$ heat wave at 5 harmonics of the modulation frequency for discharge 17176. $\mathrm{t}=3.6-4 \mathrm{~s}$. From highest to lowest amplitudes: $1^{\text {st }}$ to $5^{\text {th }}$ harmonic.

\section{Transport analysis and modelling}

Full transport simulations of steady-state and modulation data for the shots described in Sect.3 have been performed using the ASTRA code [17] and various empirical transport models featuring both diffusion and convection with adjustable values to fit the experimental data. The results are discussed in this section, starting with the interpretative power balance analysis. Some simulations using the first principle based Weiland model [4] are described in Sect.5.

\subsection{Power balance}

The power balance in the core plasma where there is no ECH power is quite delicate, as the Ohmic source is almost equally balanced by the sink due to electron-ion coupling, so that the electron heat flux is close to zero. Fig.10a illustrates for shot 17175 the different power terms, whilst Fig.10b shows the resulting power balance electron heat diffusiv- 
ity $\chi^{\mathrm{PB}}$. The Ohmic power and the electron-ion collision term have been calculated from ASTRA simulations matching the available experimental information. The $T_{i}$ profile has been simulated assuming for the ion heat diffusivity an empirical parabolic type of profile, whose coefficients are adjusted to match the measured central $T_{i}$ value and also an indication on the $\mathrm{T}_{\mathrm{i}}$ at mid-radius derived from measurements in other shots with similar characteristics. The profile of the effective charge $\left(Z_{\text {eff }}\right)$ is assumed growing towards the edge, according to spectroscopic indications in other AUG shots similar to this one, but in any case it has been checked that this choice does not influence the power balance much; the absolute value of $Z_{\text {eff }}$ is adjusted by matching the measured loop voltage. The absolute value of $T_{i}$ and its profile shape, which determine the e-i sink profile, are instead crucial in determining the sign of $\chi^{\mathrm{PB}}$ in the region $0.3<\rho_{\mathrm{t}}<0.5 ; \chi^{\mathrm{PB}}$ in the region $0<\rho_{\mathrm{t}}<0.3 \chi^{\mathrm{PB}}$ is always close to 0 . One can see in Fig.10a and $\mathrm{b}$ that for the measured value $T_{\mathrm{i} 0} \sim 900 \mathrm{eV}$ within experimental uncertainties $\chi^{\mathrm{PB}}$ is negative for $0.3<\rho_{\mathrm{t}}<0.5$, for lower values of $T_{\mathrm{i} 0}$ $(\sim 700 \mathrm{eV})$ it becomes even more negative but for higher values of $T_{\mathrm{i} 0}(\sim 1100 \mathrm{eV})$ it becomes close to 0 or positive. Such value of $T_{i}$ is still within the $\pm 20 \%$ uncertainty in $T_{i}$, although too close to $T_{e}$ for such low density electron heated discharge. The radiated power is measured to be very low in these experiments, but in these low power conditions profile measurements are not very accurate, so a uniform level $\mathrm{P}_{\mathrm{rad}} \sim 0.005 \mathrm{MW} / \mathrm{m}^{3}$ has been assumed as reasonable estimate for the core region. The ECH power profile has been optimized to achieve a satisfactory reproduction of modulation data and is slightly broader than predicted by beam tracing. This is coherent with other studies in ASDEX Upgrade [18]. Its influence on power balance is not important inside $\rho_{\text {tor }}=0.5$. Core stray power deposition can be excluded as discussed in Sect.2. In conclusion, we can state that in shot 17175 the electron heat flux is very small in the core, but its sign cannot be unambiguously determined due to various sources of uncertainties in measurements.
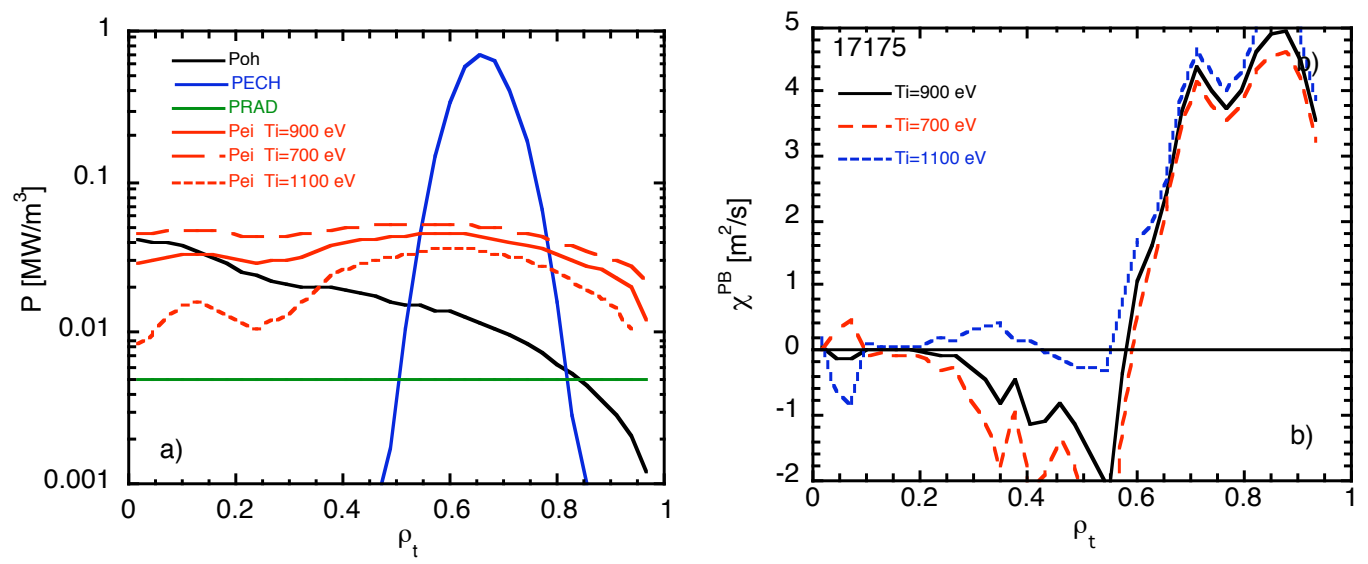

Figure 10. Power balance for shot 17175 (low density) during the steady-state phase ( $t=3.2-4 \mathrm{~s})$. a) Estimates of the different terms of the power balance: $\mathrm{P}_{\mathrm{OH}}$ and $\mathrm{P}_{\mathrm{ECH}}$ are source terms and $\mathrm{P}_{\mathrm{ei}}$ and $P_{\text {rad }}$ are sinks. $P_{e i}$ is estimated for 3 different values of $T_{i 0}$, the measured one being $T_{i 0}=900 \mathrm{eV} . b$ ) Profiles of power balance electron heat diffusivity for 3 different values of $\mathrm{T}_{\mathrm{i} 0}$.

Power balance analysis of the 3 shots presented in Sect. 3 using the measured $T_{i}$ and a $Z_{\text {eff }}$ profile growing towards the edge (matching the measured loop voltage) is presented in Fig.11. All the simulations presented in this paper will be performed under these conditions unless otherwise specified. Shot 17179 is similar to 17175 , with a somewhat negative $\chi_{\mathrm{e}}$ in the region $0.4<\rho_{\mathrm{t}}<0.6$, and the same caveats discussed for 17175 . In shot 17176 
(high density) the situation is instead rather different because, even if the heat flux is even more negative due to the stronger e-i sink, the

$\mathrm{T}_{\mathrm{e}}$ gradient reverses its sign in the region $0.4<\rho_{\mathrm{t}}<0.6$, so the resulting $\chi^{\mathrm{PB}}$ is positive there.

We conclude that of the shots analyzed, only the low density ones present some indications based on power balance in favour of a convective component in the heat flux in the region $0.4<\rho_{\mathrm{t}}<0.6$, although the uncertainties do not allow to regard this as a stringent proof. This region $0.4<\rho_{\mathrm{t}}<0.6$ is therefore the one where our attention will be most focused in the following regarding the possible existence of heat convection.

\subsection{The effect of a heat pinch on modulation data}

Given the uncertainties associated with the sign of the power balance in these conditions of very low heat flux, the information coming from modulation becomes important to progress further in the heat pinch investigation. In this section we describe with didactic purposes the effect of a heat pinch term on the modulation data using for sake of simplicity a constant parabolic $\chi_{\mathrm{e}}$ profile as in Fig.12a. The heat pinch (U) is modelled as a constant profile with Gaussian shape peaked just inside $\rho_{\text {dep }}$ as in Fig.12a. The simulations are made for the reference shot 17175. Temperature profiles are shown in Fig.12b and amplitude $\left(1^{\text {st }}\right.$ and $3^{\text {rd }}$ harmonic) and phase ( $1^{\text {st }}$ harmonic) profiles are shown in Fig. $12 \mathrm{c}, \mathrm{d}$ for 4 different peak values of $U$.
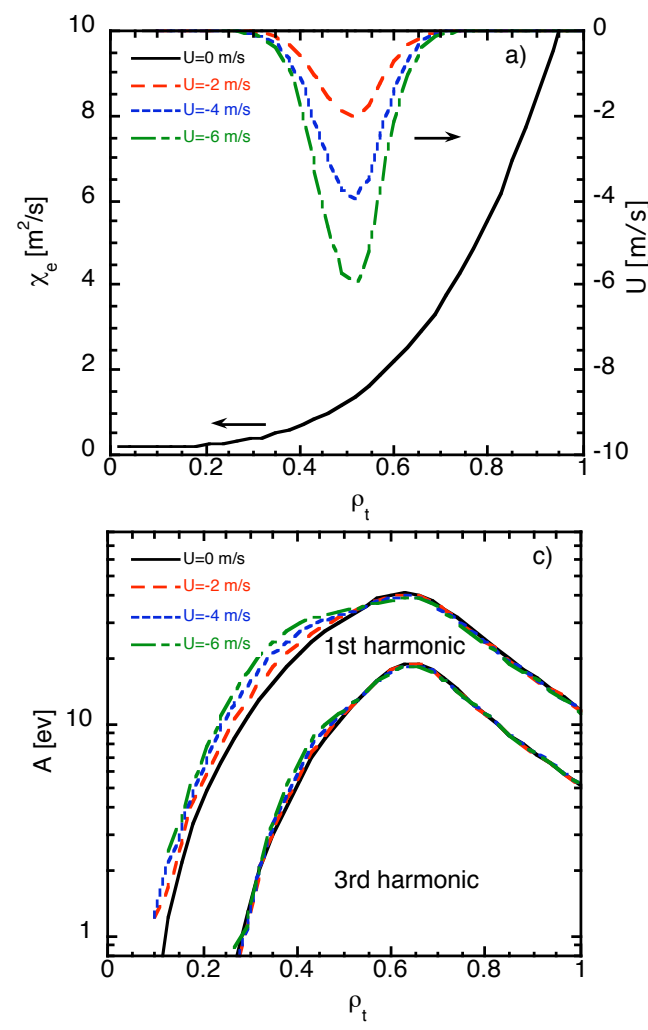

Figure 11. Power balance electron heat diffusivitu for the 3 shots desyiged in

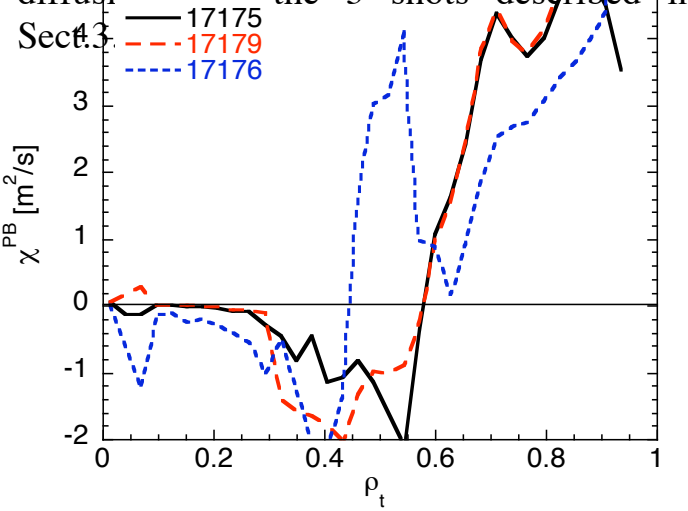


Fig.12 that a heat pinch affects only the $\mathrm{T}_{\mathrm{e}}$ and $\mathrm{A}$ profiles, whilst the $\varphi$ profiles are not sensitive to $U$ and are basically determined by the $\chi_{\mathrm{e}}$ profile. The $\chi_{\mathrm{e}}$ profile in Fig. $12 \mathrm{a}$ has been in fact chosen to fit the $\varphi$ profiles of shot 17175. The pinch acts in the direction of peaking the $T_{\mathrm{e}}$ profiles and inflating the $A$ profile inside $\rho_{\text {dep }}$ at the lowest frequencies, creating a region of flatter amplitudes yielding $\chi^{\mathrm{A}}>\chi^{\varphi}$, as indeed observed in the data. The effect of $U$ on amplitudes vanishes at higher frequencies.

In order to reproduce the amplitude features just with a pinch within this simple constant $\chi_{\mathrm{e}}$ model, values $U \sim-4 \mathrm{~m} / \mathrm{s}$ would be required, which however would produce a too high peaking of the $\mathrm{T}_{\mathrm{e}}$ profile compared to experiment. We will therefore introduce in the following section a more complex model for $\chi_{\mathrm{e}}$ based on the critical gradient concept.

\subsection{Simulations using an empirical critical gradient model plus a heat pinch term}

The simulations with constant $\chi_{\text {e }}$ presented in Sect..4.2 were made only for a didactic purpose, because it is in fact by now well known that such purely diffusive models are not able to explain the large amount of experimental results obtained in the last years in the field of electron transport. Most of such results have in fact successfully been explained on the basis of a critical gradient concept as mentioned in the Introduction [9-15]. Starting from the basic theoretical result that ITG/TEM turbulence arises when critical values for $\mathrm{R} / \mathrm{L}_{\mathrm{Te}}$ (for TEM) or $\mathrm{R} / \mathrm{L}_{\mathrm{Ti}}$ (for ITG) are exceeded, a simple semi-empirical model for the electron heat diffusivity has been proposed [9] and successfully used for the interpretation of $\mathrm{T}_{\mathrm{e}}$ modulation experiments in several machines $[9,11,12,19]$. This model, which we will call in the following CGM (Critical Gradient Model), features a $\chi_{\mathrm{e}}$ profile of the form

$$
\chi_{e}=\chi_{s} q^{1.5} \frac{T_{e}}{e B} \frac{\rho_{s}}{R}\left(\frac{R}{L_{T_{e}}}-\kappa_{c}\right) H\left(\frac{R}{L_{T_{e}}}-\kappa_{c}\right)+\chi_{0}(\rho)
$$

where $\rho_{\mathrm{s}}=\sqrt{m_{i} T_{e}} / e B, \mathrm{q}$ is the safety factor, $\chi_{0}$ yields the level of residual transport (not necessarily neoclassical), $\chi_{\mathrm{s}}$ is a dimensionless number giving the strength of the turbulent transport term assuming a gyro-Bohm normalization, and $\kappa_{\mathrm{c}}$ is the threshold. $\mathrm{H}$ is the Heaviside function. $\chi_{\mathrm{e}}$ is determined by whether the actual value of $R / L_{\mathrm{T}}$ is below or above a critical threshold value $\kappa_{\mathrm{c}}$, and by how strongly the plasma turbulence reacts when the threshold is exceeded (this gives the "stiffness level" of the model, expressed by $\chi_{s}$ ).

It is therefore mandatory to explore the heat pinch issue using the same CGM model that is required to explain the various electron transport results obtained in other AUG experiments. To the diffusive component in Eq.(3) in the present work the possibility of a heat convection component has been added, constant in time and with a similar spatial profile peaked just inside $\rho_{\text {dep }}$ as used above (Fig.12).

It turns out that simulations of the AUG shots described in Setc. 3 with the GGM model plus heat pinch term are rather delicate because of the non-linear interaction between the pinch term which acts on the value of $\mathrm{R} / \mathrm{L}_{\mathrm{Te}}$ and the threshold mechanism which is regulated by the value of $\mathrm{R} / \mathrm{L}_{\mathrm{Te}}$. Since in the region outside $\rho_{\text {dep }}$ the plasma is always above threshold due to the large heat flux, basically 3 possible situations for the core region can be distinguished in the simulation scenario:

1) the core region inside $\rho_{\text {dep }}$ is always below threshold, in which case the simulation of the core falls back to the constant $\chi_{\mathrm{s}}$ model described in Sect.4.2. In this case the heat wave propagation is determined by $\chi_{\mathrm{e}}^{\mathrm{hp}}=\chi_{0}$ plus a possible convection term.

2) the core region inside $\rho_{\text {dep }}$ is always above threshold apart from the very core where the plasma eventually goes below threshold [19]. In this case the heat wave propagation is determined by 


$$
\chi_{e}^{h p}=\chi_{s} \mathrm{q}^{1.5} \frac{T_{e}}{e B} \frac{\rho_{s}}{R}\left(2 \frac{R}{L_{T_{e}}}-\kappa_{c}\right)+\chi_{0}(\rho)
$$

plus a possible convection term. The wave propagation can then be much faster and the amplitude will have a much slower decay (flatter A and $\varphi$ profiles) if the stiffness level $\chi_{\mathrm{s}}$ is high. We note that in this condition, as pointed out in [9], an apparent convective term due to the $\mathrm{T}_{\mathrm{e}}^{3 / 2}$ dependence of $\chi_{\mathrm{e}}$ can also become visible on the modulation data.

3) The core region inside $\rho_{\text {dep }}$ (or a part of it) is close to threshold and oscillates from below to above threshold due to the ECH modulation. As a result $\chi_{e}$ and even more $\chi_{e}^{\text {hp }}$ oscillate between a low and a high level. This can give rise to significant distortions in the shape of the $\mathrm{A}$ and $\varphi$ profiles.

The experimental profile of $\mathrm{R} / \mathrm{L}_{\mathrm{Te}}$ for shot 17175 is shown in Fig.13. Taking a spatially uniform threshold, values of $\kappa_{\mathrm{c}}$ in the range 3-7 are normally found in AUG modulated shots with less off-axis power deposition and within a range of q values [19]. In the following studies a spatially dependent threshold will be used as suggested by the theory of TEM modes [4], whose threshold increases towards the plasma axis due to the reduction of the fraction of trapped electrons. Its profile has been calculated using the expression given in [21]

$$
\kappa_{c}=\frac{4}{3 \varepsilon_{n}}+\frac{20}{9 \Gamma}+\frac{\Gamma}{2}\left(1-\frac{1}{\varepsilon_{n}}\right)^{2}
$$

where $\Gamma=f_{t} /\left(1-f_{t}\right)\left(f_{t}\right.$ is the fraction of trapped particles $)$ and $\varepsilon_{n}$ is the ratio of the drift and diamagnetic frequencies $\varepsilon_{\mathrm{n}}=\omega_{\mathrm{De}} / \omega_{* \mathrm{e}}$.

The threshold profile from Eq.5 is shown in Fig.13 for shot 17175. From Fig.13 one can see that the likely situation in the region inside $\rho_{\text {dep }}$ for shots 17175 and 17179 is the one corresponding to option 3) (oscillating around the threshold), whilst for shot 17176 the region $0.3<\rho_{t}<0.6$ with flat or hollow $\mathrm{T}_{\mathrm{e}}$ profile corresponds to option 1) (always below threshold), also due to the fact that the TEM threshold is increased by the stabilizing effect of collisionality on TEM [13]. In the simulations a multiplying coefficient has been used in front of Eq. 5 to adjust the threshold absolute magnitude to reproduce the data. The best value for such coefficient has been found to be around 0.5 for all 3 shots.

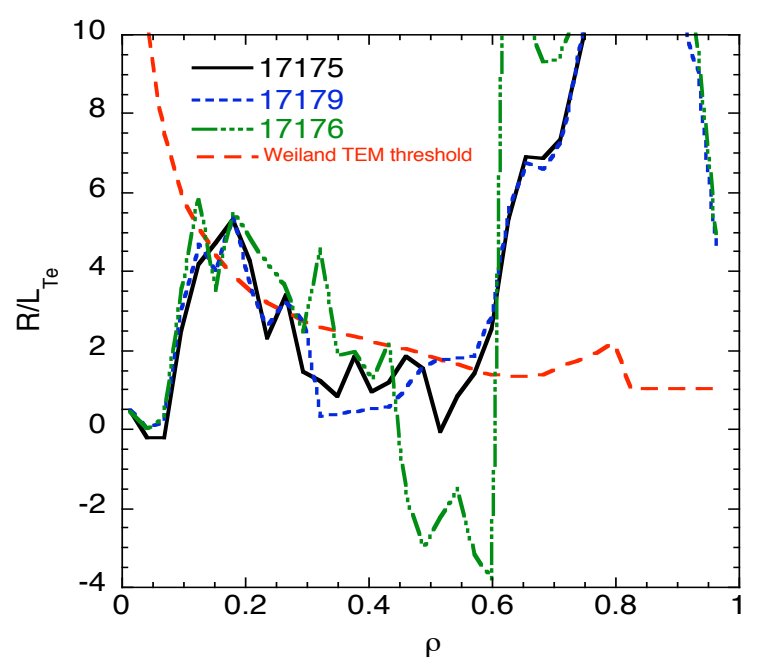

Figure 13. Experimental time averaged $\mathrm{R} / \mathrm{L}_{\mathrm{Te}}$ profiles for shots $17175,17179,17176$ in the same time intervals as in Figs,4,6,8. The threshold for TEM calculated for shot 17175 from Eq.5 is also plotted.

\subsubsection{Simulation of low density, high modulation amplitude case}

A reasonable reproduction of the data of 17175 has been obtained using the CGM model in conditions 3), i.e. with the $\mathrm{T}_{\mathrm{e}}$ profile in the region $\rho<\rho_{\text {dep }}$ close to threshold and oscillating around it during the modulation cycle. Fig. 14 illustrates the simulated $\mathrm{T}_{\mathrm{e}}$ and $\mathrm{A}$ and $\varphi$ profiles and compares them with the data. In Fig. 15 the oscillation around the 
threshold is illustrated in detail: in a) the threshold used in the simulation and the simulated $\mathrm{R} / \mathrm{L}_{\mathrm{Te}}$ profiles at various times during the modulation cycle are shown, and in $\mathrm{b}$ ) the resulting $\chi_{\mathrm{e}}$ profiles. Inside $\rho_{\mathrm{tor}}=0.3$ the plasma drops below threshold, which gives rise to the change in amplitude slope as seen in the data. The combination of a $U=-1 \mathrm{~m} / \mathrm{s}$ and the threshold mechanism allows to reproduce well the experimental profiles in the region $0.3<\rho_{\text {tot }}<0.6$. It is important to note that the amplitude distortion is now produced not by the direct effect of $U$ (which is too small to give an appreciable effect) but by the oscillation of the $T_{e}$ profile around threshold, which produces large oscillations in $\chi_{e}$ and $\chi_{e}{ }^{h p}$. In this way it is possible to obtain a good reproduction both of steady-state and modulation data. The role of $U$ in this case is that of keeping the $T_{e}$ profile peaked enough to be close to threshold, and not below it, so allowing the oscillation around threshold which yields the amplitude distortion. Eliminating the $\mathrm{U}$ term leads to a bad reproduction of the data in the region inside $\rho_{\text {dep }}$, as shown in Fig. 16, because in this case the $\mathrm{T}_{\mathrm{e}}$ gradient drops below threshold and a low constant $\chi_{\mathrm{e}}$ appears which produces a strong damping of the A profile. We also note that in this sense the presence of a convective term is not strictly implied by the data, as any alternative mechanism resulting in maintaining the $T_{e}$ peaking close to threshold would produce a similar effect. For example similar results can be obtained by assuming a higher $\mathrm{T}_{\mathrm{i}}$ than measured $\left(\mathrm{T}_{\mathrm{i} 0} \sim 1100 \mathrm{eV}\right)$ in order to have a more positive power balance that keeps the $\mathrm{T}_{\mathrm{e}}$ gradient high enough.

Attempts to reproduce the data under conditions 1) or 2) do not yield good results. In fact option 1) is equivalent to the constant $\chi_{e}$ model already discussed in Sect.4.2, whilst in option 2) the resulting $\chi_{\mathrm{e}}$ (always above threshold) is higher and requires more convection to reproduce the $\mathrm{T}_{\mathrm{e}}$ peaking, thereby yielding too flat A profiles.

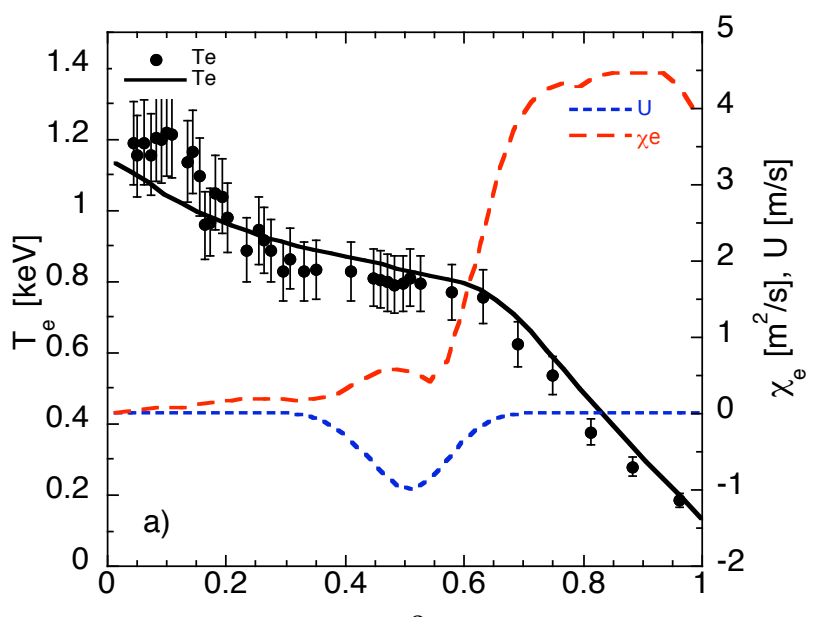

Figure 14. Experimental (symbols) and simulated (lines) profiles of $\mathrm{T}_{\mathrm{e}}$ (a), amplitudes (b) and phases (c) for shot 17175 (low density, high modulation amplitude) using the model in Eq.4 with $\chi_{s}=0.12$. In a) also the $\mathrm{U}$ profile and the $\chi_{\mathrm{e}}$ profile at one time well in the ON phase are shown.
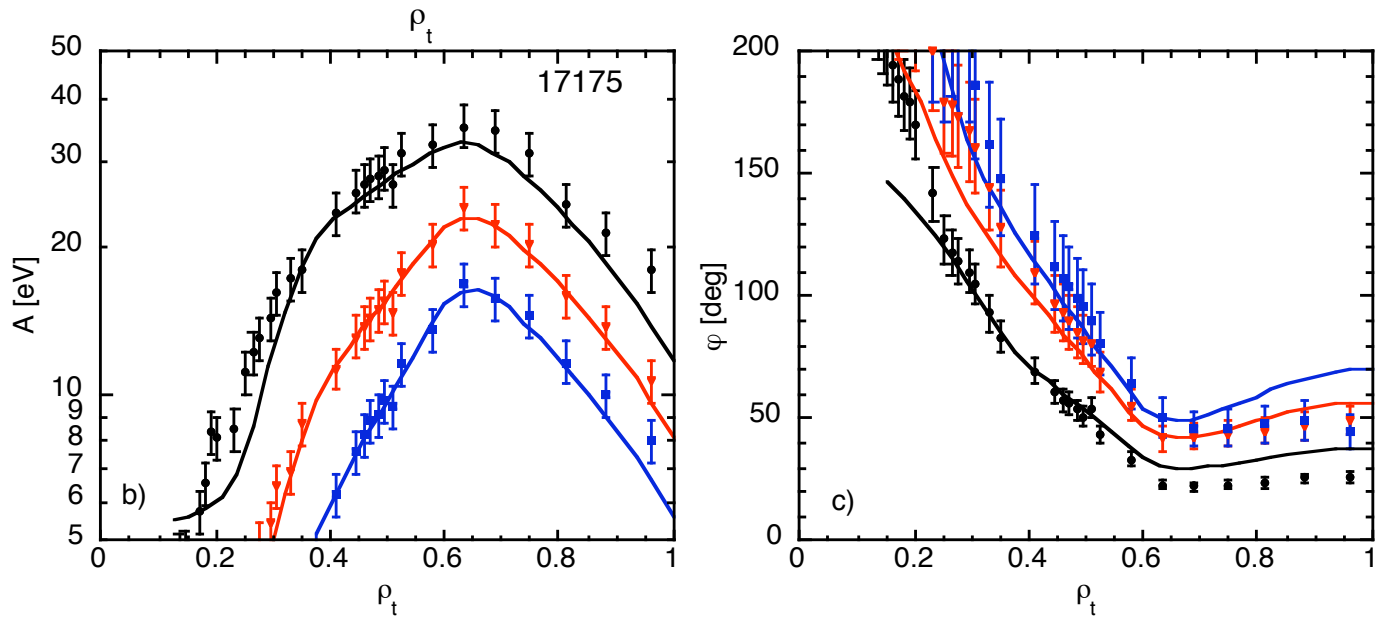

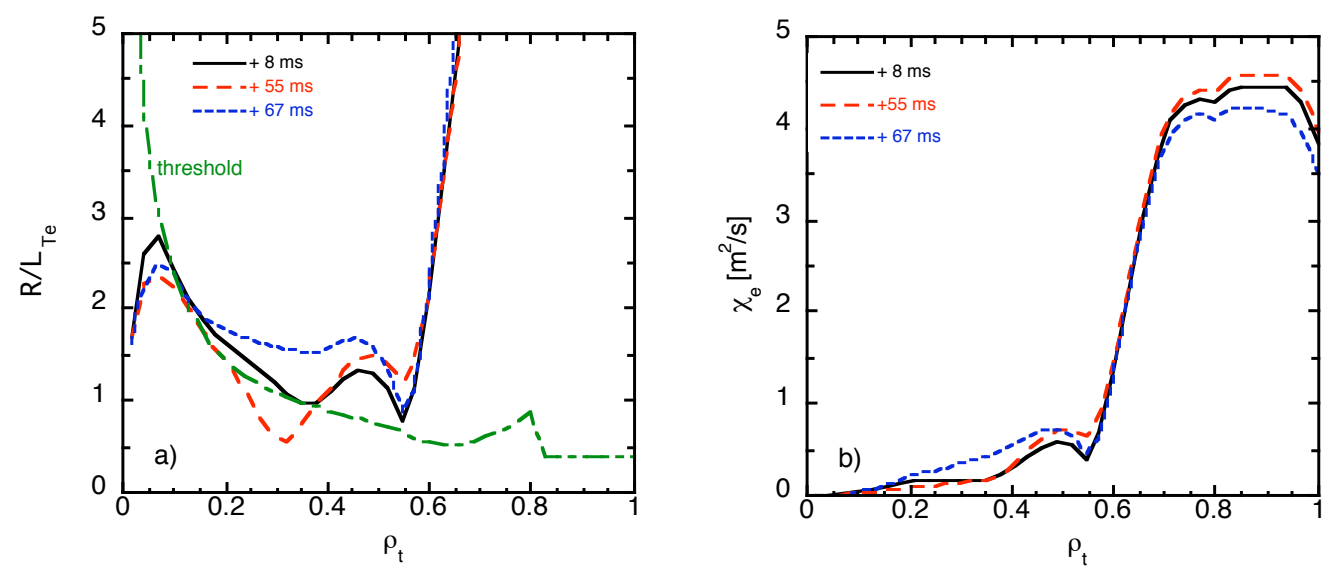

Figure 15. a) threshold and simulated profile of $R / \mathrm{L}_{\mathrm{Te}}$ at various times during the cycle (with respect to power switch on time) for the simulation of Fig.14; b) resulting $\chi_{e}$ profile at the same times. We remind that a cycle in 17175 consists of $60 \mathrm{~ms}$ ON and $8 \mathrm{~ms}$ OFF.

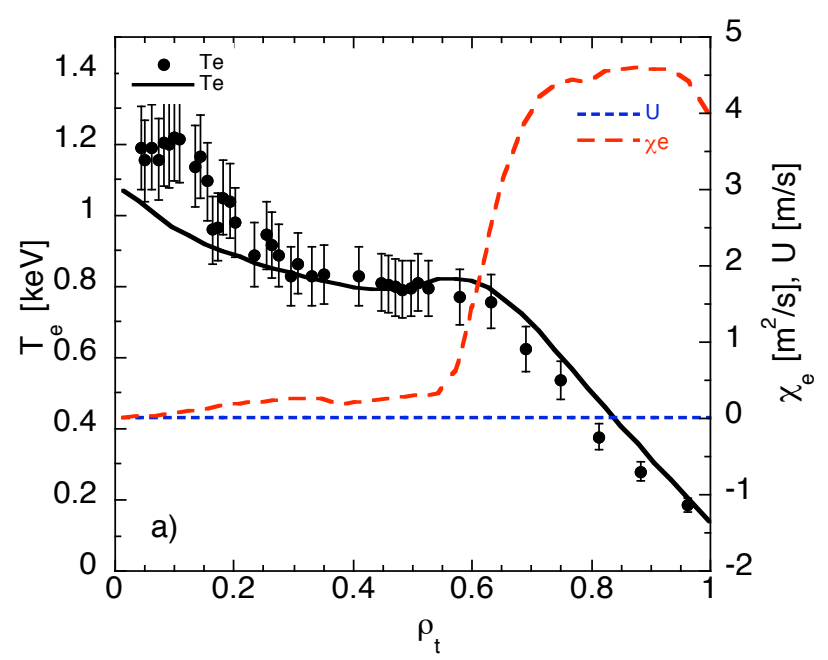

Figure 16. same as in Fig.14 but with $\mathrm{U}=0 \mathrm{~m} / \mathrm{s}$.
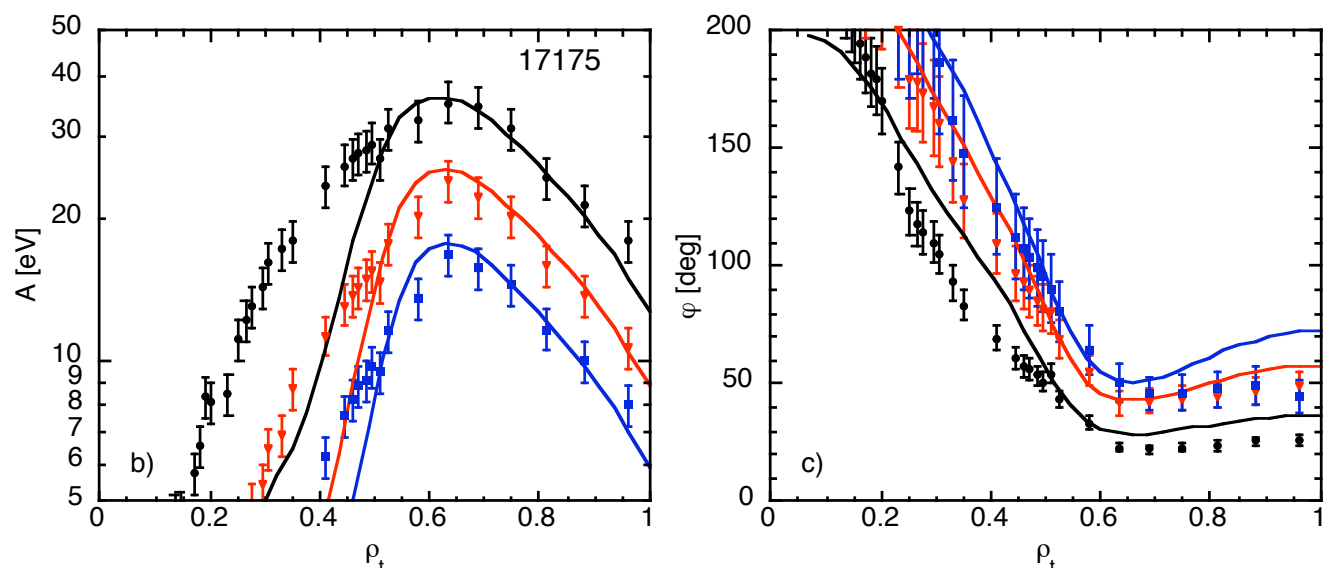

\subsubsection{Simulation of low density, low modulation amplitude case}

The simulation of shot 17179 is particularly demanding because in this shot a distortion of the amplitude profile is visible both on $1^{\text {st }}$ and $3^{\text {rd }}$ harmonics and, in addition, the amplitudes are not just flat but even increasing inside $\rho_{\text {dep }}$, so that the maximum is found well inside $\rho_{\text {dep }}$, whilst the phase minimum stays at $\rho_{\text {dep }}$ with a regular profile behaviour. As far as we are aware, there is no other mechanism than heat convection (real or effective, i.e. for example coming from a $\mathrm{T}_{\mathrm{e}}$ dependence or a strong spatial dependence of $\chi_{\mathrm{e}}[16]$ ) that 
can cause amplitudes to grow away from the deposition radius whilst leaving phase profiles unaffected. However the convective-like effects due to the $\mathrm{T}_{\mathrm{e}}^{3 / 2}$ or spatial dependence of $\chi_{\mathrm{e}}$ in Eq.(4) (and implicitly already contained in all our simulations) do not seem to be important in the conditions required to simulate these discharges. Therefore within the framework of this model and unless very peculiar $\chi_{\mathrm{e}}$ dependences are invoked, the introduction of real convection into the simulation becomes a necessity. This is why we regard shot 17179 as the one providing evidence of the existence of convection in this series of shots. Implicitly, the findings for 17179 support the use we made of a small convective term also for the simulation of 17175, although evidence for it was not as strict in 17175 , as discussed above. The best simulation of 17179 obtained with the CGM model of Eq.(4) plus a pinch is shown in Fig.17. It is not optimum but finer tuning of the complex interaction between CGM and pinch mechanisms is very delicate and not essential to the demonstration that it is a suitable blend of heat pinch profile, threshold profile, and stiffness factor that leads to the possibility of reproducing these very peculiar experimental profiles. In this respect the modeling work should now continue based on first principle modelling, to gain more physical insight into these results. To trigger such work is one of the objects of the present paper. One important thing to note from Fig. 17 is that a larger and broader $\mathrm{U}$ is needed here to provide the right shape of the A profile, and such convective term needs to stay in a region above threshold in order to minimize (due to profile stiffness) its effect on the simulated $T_{e}$ profile. On the other hand, the effect of modulation of the $T_{e}$ profile around the threshold is smaller in this discharge because the amplitude modulation is much smaller. This is partly compensated by a slightly higher stiffness level $\left(\chi_{s}=0.2\right)$. The effect of threshold turns anyway to be an essential ingredient to reproduce the change of slopes of amplitudes around $\rho_{\mathrm{tot}}=0.4$, visible also on phases.

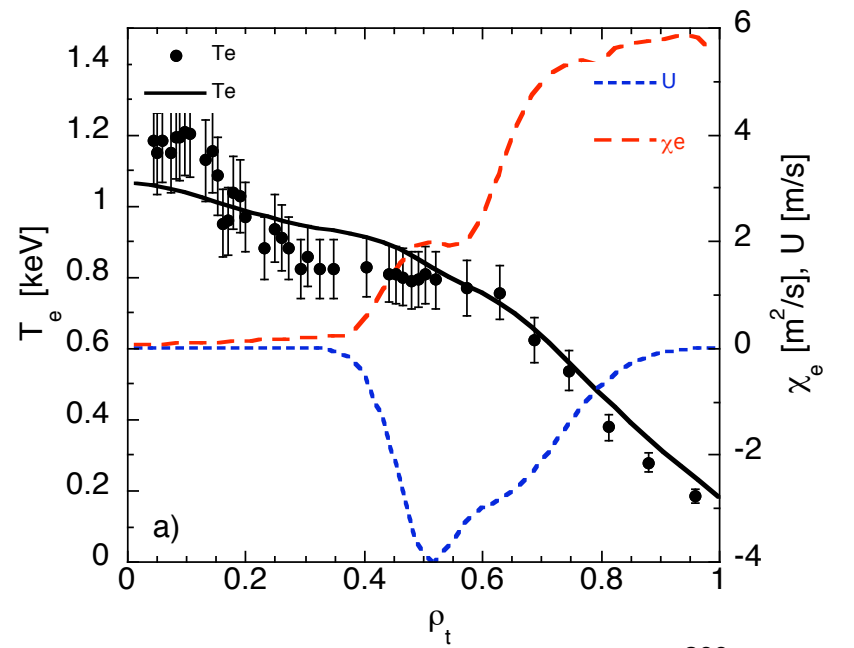

Figure 17. Experimental (symbols) and simulated (lines) profiles of $\mathrm{T}_{\mathrm{e}}(\mathrm{a})$, amplitudes (b) and phases (c) for shot 17179 (low density, small modulation amplitude) using the model in Eq.4 with $\chi_{\mathrm{s}}=0.2$. In a) also the $U$ profile and the $\chi_{\mathrm{e}}$ profile at one time well in the ON phase are shown.
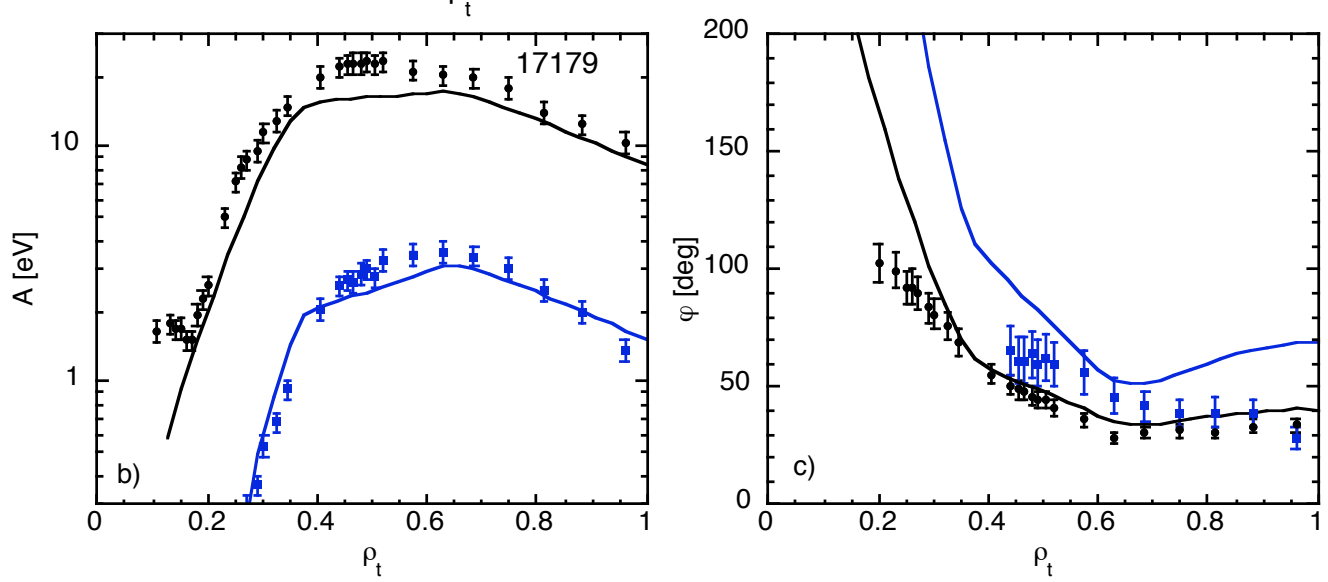


\subsubsection{Simulation of high density case}

Simulation of the high density shot 17176 is simpler because, as discussed in Sect.3.2, amplitudes do not show the distortion from diffusive behaviour just inside $\rho_{\text {dep }}$ seen in shot 17175 and 17179. Also, the $T_{\text {e }}$ profile shows a hollow region just inside $\rho_{\text {dep }}$, as expected from diffusive transport in the presence of a negative heat flux. Reproduction of the main features in the data that differ from low density cases, i.e. the inversion of the $\mathrm{T}_{\mathrm{e}}$ gradient and the sharp drop of amplitudes inside $\rho_{\text {dep }}$, has been achieved with the CGM model in conditions where the whole region inside $\rho_{\text {dep }}$ is below threshold. No convection is required. Fig. 18 illustrates the results. We remark here that in all our simulations quantitative matching of the peaking of $\mathrm{T}_{\mathrm{e}}$ in the very core region (just due to the balance between the low value of heat diffusivity and the residual positive power source) is not so important for the aim of this paper, which focusses on the existence of convection in the region inside $\rho_{\text {dep }}$ up to $\rho \sim 0.3$. In this sense it is also acceptable that the simulation does not fit the local flattening of both amplitudes and phases around $\rho_{\text {tot }} \sim 0.35$, which, clearly involving also phases, is of different nature than the convective distortion addressed in this paper, and is possibly due to the presence of an MHD island at that location.

This discharges is a nice confirmation that the effects described in the previous sections for the low density cases are real plasma effects and not due to any peculiar ECH deposition effect, because otherwise they would not vanish for such a small change in plasma density (from 2.7 to $3.410^{19} \mathrm{~m}^{-3}$ ). It is also a nice confirmation of the role played by the critical gradient threshold, which can generate such a different transport behaviour for shots differing only by a small amount of density, but enough to pass from a situation above or close to threshold to a situation clearly below threshold.

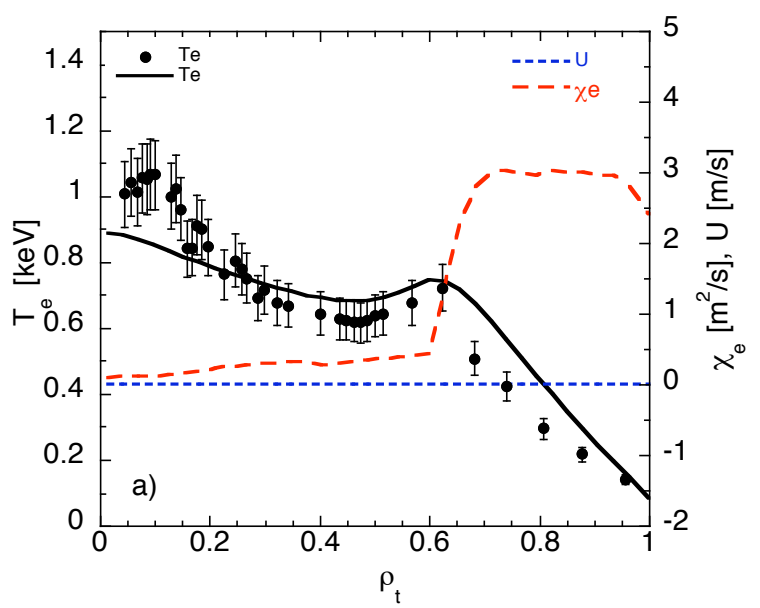

Fig.18. Experimental (symbols) and simulated (lines) profiles of $T_{e}(a)$, amplitudes (b) and phases (c) for shot 17176 (high density) using the model in Eq.4 with $\chi_{\mathrm{s}}=0.12$ and $\mathrm{U}=0 \mathrm{~m} / \mathrm{s}$. In a) also the $\mathrm{U}$ profile and the $\chi_{\mathrm{e}}$ profile at one time well in the ON phase are shown.
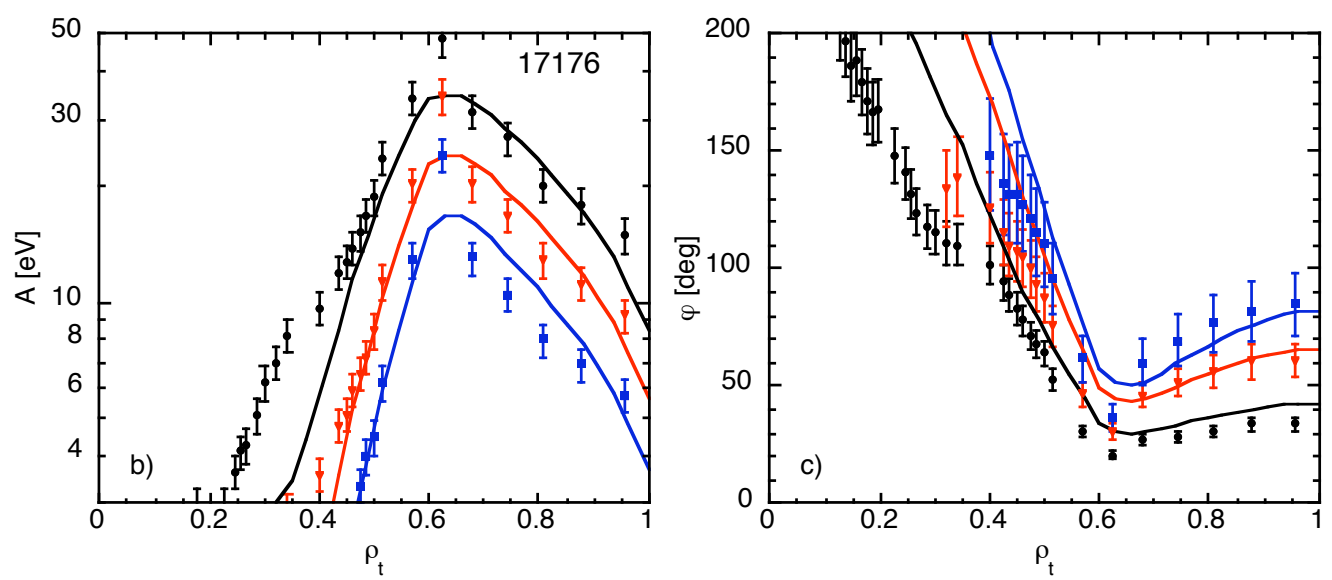


\subsection{The switch-on phase}

As a final point we come back to the $\mathrm{T}_{\mathrm{e}}$ evolution at ECH switch-on, discussed in Sect.3.1 (Fig.1 and 2a). A simulation of shot 17175 full time evolution using the critical gradient model for $\chi_{\mathrm{e}}$ and $\mathrm{U}=-1 \mathrm{~m} / \mathrm{s}$ with the same choice of parameters as the simulation in Fig.14 is shown in Fig.19a. The current profile is evolved assuming neo-classical resistivity and taking into account the bootstrap current according to [22]. The broadening of the current profile following off-axis heating causes the decrease on central Ohmic power that leads to the slow decrease of $\mathrm{T}_{\mathrm{e}}$ in the core channels, consistent with experiment. However it is clear that the increase in central $\mathrm{T}_{\mathrm{e}}$ at $\mathrm{ECH}$ switch-on is largely underestimated with respect to experiment. In fact this simulation gives an indication of how peculiar the experimental evidence shown in Fig. 1 is. In order to increase the central $T_{e}$ response, it is necessary to increase the value of $U$ and broaden its profile with respect to Fig.14. An example of the obtained time evolution with $U=-3 \mathrm{~m} / \mathrm{s}$ (and broader profile) is shown in Fig.19b. This suggests that during the big initial transient the transport parameters are different and evolving in time, and a larger convective flow may be transiently present. It is also possible that the core heat diffusivity is reduced after ECH switch-on, for example following a density flattening and consequent reduction of the TEM drive, thus contributing to the anomalous $\mathrm{T}_{\mathrm{e}}$ rise in the centre. A proper simulation of the switch-on phase is outside the scope of the present paper and would require more sophisticated models where threshold, stiffness and pinch are time-dependent following presently unknown dependencies on plasma parameter profiles.
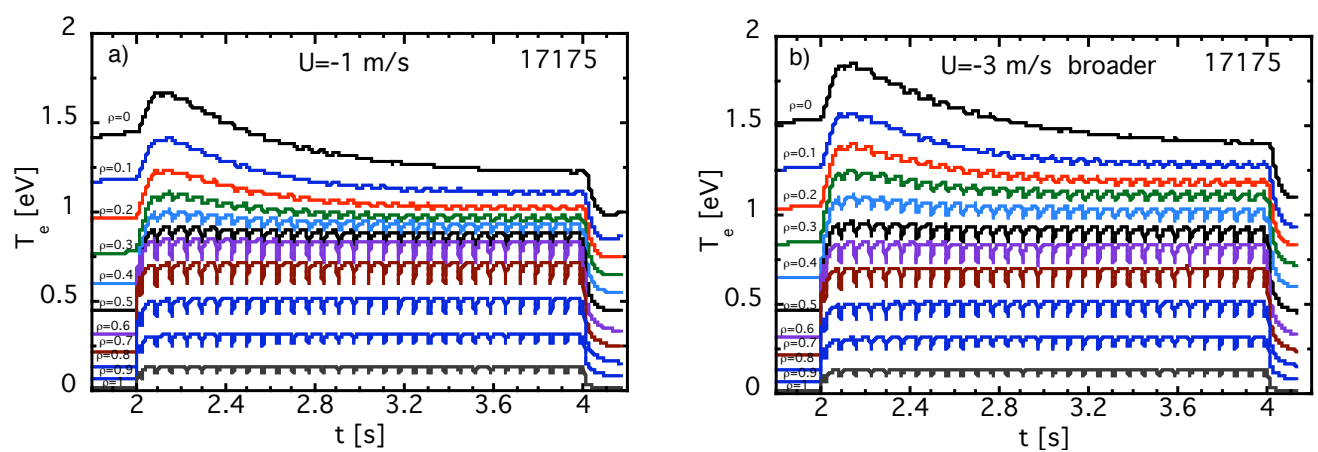

Figure 19. Simulated $T_{\mathrm{e}}$ time evolution for shot 17175 (to be compared with experimental data in Fig.1). In a) the simulations are performed using the critical gradient model for $\chi_{\mathrm{e}}$ and $U=-1 \mathrm{~m} / \mathrm{s}$ as in Fig.14, while in b) $U$ has been increased to $U=-3 \mathrm{~m} / \mathrm{s}$ and its profile broadened.

\section{First principle based modelling}

In an attempt to understand possible physical mechanisms explaining the heat pinch evidence in AUG, simulations were done for a few AUG discharges with various ECH deposition radii using the 1-D fluid Weiland model [4] including off-diagonal and convective terms. This model has been found successful in reproducing the heat pinch evidence both in DIII-D [23] and RTP [8].

Fig.20 illustrates the performance of the Weiland model in the case of AUG discharge 17175. In this simulation also the density is consistently evolved and matches well the experimental $\mathrm{n}_{\mathrm{e}}$ profile. The resulting $T_{\mathrm{e}}, A$ and $\varphi$ profiles are compared with experimental data in Fig.20. In Fig.21, the different terms of the electron heat flux predicted by the Weiland simulation are shown. One can see that a small convective term is predicted in the core region, together with a comparable inward off-diagonal flux, which helps the small Ohmic heating in maintaining the $\mathrm{T}_{\mathrm{e}}$ profile peaked in the region $\rho=0.15-0.4$. This 
peaking is mainly made possible by the extremely low value of $\chi_{\mathrm{e}}$ in this region. However such low value of $\chi_{\mathrm{e}}$ is inconsistent with modulation data, as it causes a very sharp drop of amplitudes and a steep increase of phases inside $\rho_{\text {dep }}$, very different from experimental observations. We can conclude that the Weiland model simulation, although somehow reproducing the $T_{\mathrm{e}}$ profile peaking due to the extremely low $\chi_{\mathrm{e}}$ in presence of a small positive heat flux, is absolutely incompatible with the observed modulation response, due to prediction of too small heat diffusivity and too small heat pinch. The reason of this has to do with the fact that the level of turbulence in the whole core region is low due to lack of power sources, which implies low turbulence and consequently low heat diffusivity and no generation of turbulent pinches. This condition makes it impossible to reproduce the experimental data, where, according to our results of Sect.4, there is a complex interplay between a small pinch which keeps the $\mathrm{T}_{\mathrm{e}}$ gradient high enough to stay around the critical value, with consequent presence of turbulence and higher values of $\chi_{\mathrm{e}}$. It is possible however that a bifurcation exists during the discharge time evolution between these two situations, and that the experimental observations may be better explained in the context of a time dependent simulation addressing first the Ohmic phase and then the evolution when $\mathrm{ECH}$ is switched on. This issue will be addressed in future work. It is also possible that new theoretical ideas, such as turbulence spreading into the core region, are necessary to explain the experimental observations. Unfortunately, due to the slow time scale of the modulation in AUG at the moment it is not possible to simulate multiple AUG MECH cycles with non-linear turbulence codes.

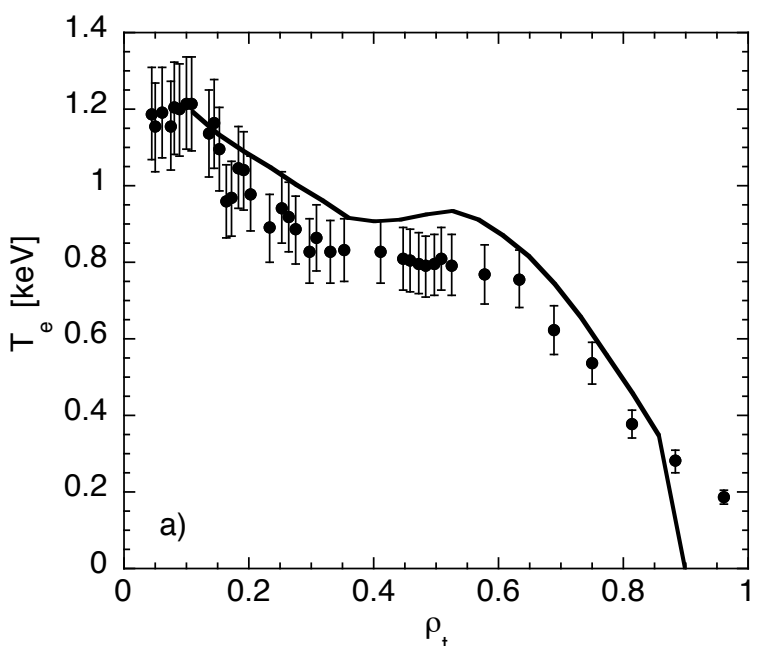

Figure 20. Experimental (symbols) and simulated (lines) profiles of a) $\mathrm{T}_{\mathrm{e}}, \mathrm{b}$ ) MECH amplitudes and c) phases at 5 harmonics for AUG discharge 17175 using the Weiland model.
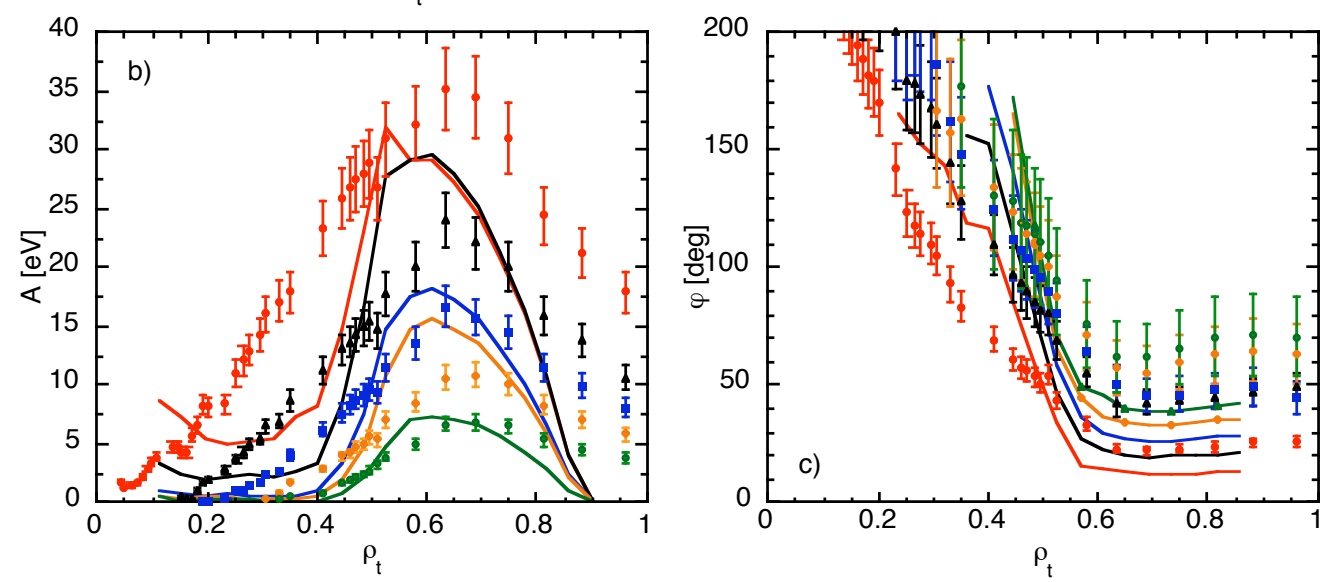


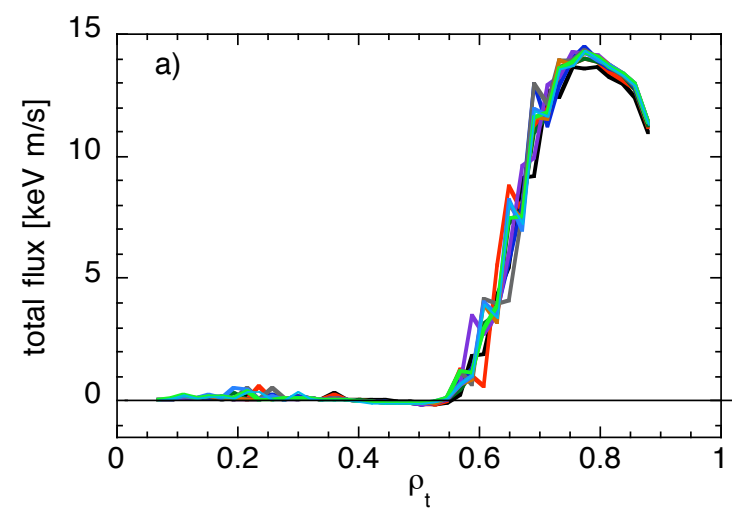

Figure 21. Total (a), off-diagonal (b) and convective (c) heat fluxes for the AUG simulation using Weiland model shown in Fig.20. Various colored traces correspond to different times during one modulation cycle.
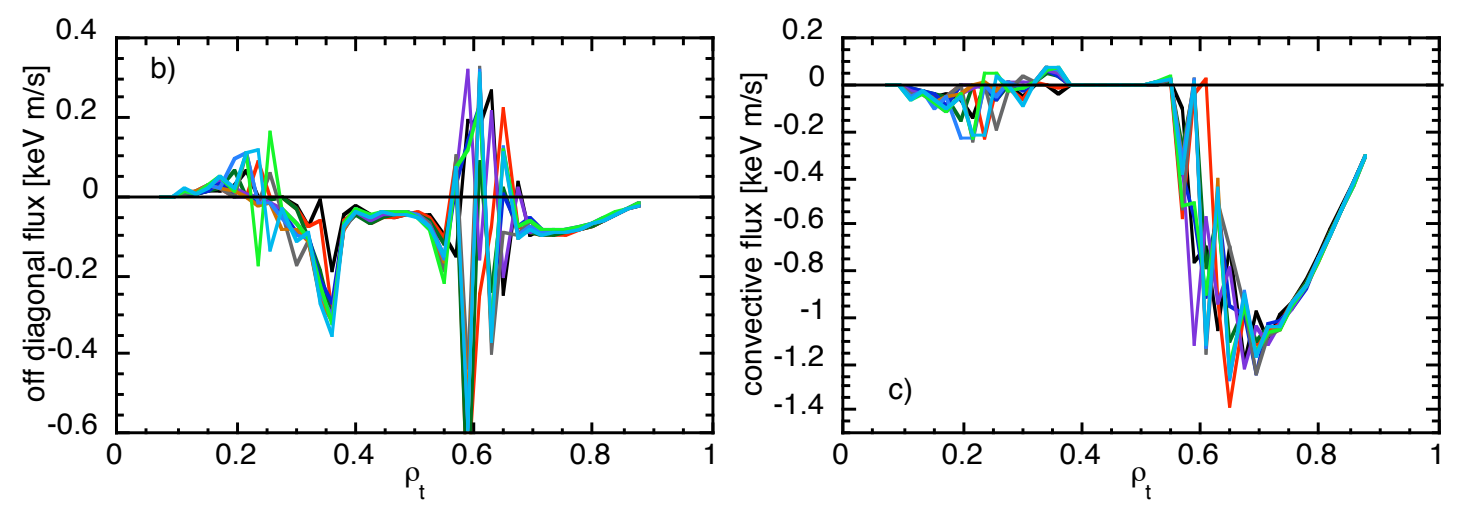

\section{Conclusions}

Modulated ECH experiments on AUG with off-axis deposition $\left(\rho_{\text {dep }} \sim 0.6\right)$ provide new type of evidence of the combined effect of a critical gradient regulated electron transport and an electron heat pinch term. At low density, where the $T_{e}$ profile is peaked in presence of a power balance close to zero and the low harmonics amplitudes of the modulation show a distortion inside the deposition radius, the data can be reproduced with an empirical transport model featuring a threshold on the inverse critical gradient length and a pinch velocity $\mathrm{U} \sim 1-3 \mathrm{~m} / \mathrm{s}$ in the region $0.3<\rho_{\text {tor }}<0.6$. In these discharges the small pinch term allows the $\mathrm{T}_{\mathrm{e}}$ profile to stay above or close to threshold and oscillate around it, giving rise to the peculiar distortion in the amplitude data measured experimentally. The need for the small pinch component arises essentially from the observation in small modulation amplitude cases that the heat wave amplitude grows whilst propagating away from ECH deposition. Power balance, although negative in the region $0.3<\rho_{\text {tor }}<0.6$, cannot be regarded as a strict proof for the existence of convection, given the experimental uncertainties. At high density, no pinch term is required to simulate the data, and the $T_{e}$ profile inside $\rho_{\text {dep }}$ is always below threshold, yielding very different $T_{e}$ and amplitude and phase profiles with respect to the low density case, and consistent with purely diffusive transport. The Weiland model has also been used to simulated the low density data, but although predicting the existence of turbulence generated heat pinches, does not reproduce well the AUG data due to too low $\chi_{e}$ value and too weak heat pinch in the core region, resulting from lack of significant core power source and associated reduced turbulent levels.

\section{Acknowledgements}

We acknowledge valuable discussions with X.Garbet, C.Angioni and A. Thyagaraja on turbulence driven pinches. 


\section{References}

[1] T.C.Luce et al., Phys. Rev. Lett. 68 (1992) 52

[2] P.Mantica et al., Phys. Rev. Lett. 85 (2000) 4534

[3] C.Sozzi et al., $18^{\text {th }}$ IAEA Fusion Energy Conference, Sorrento (2000) exp 5-13

[4] J. Weiland, "Collective modes in inhomogeneous plasma", Kinetic and Advanced Fluid Theory, IoP Publishing, Bristol and Philadelphia 2000

[5] C.Angioni et al., Phys. Rev. Lett. 90 (2003) 205003

[6] X.Garbet et al., Phys. Rev. Lett. 91 (2003) 035001

[7] X.Garbet et al., Phys. Plasmas 12 (2005) 082511

[8] P.Mantica, Phys. Rev. Lett. 95 (2005) 185002

[9] F. Imbeaux et al., Plasma Phys. Control. Fusion 43 (2001) 1503

[10] F.Ryter et al., Phys. Rev. Lett. 86 (2001) 2325

[11] F.Ryter et al., Nucl.Fusion 43 (2003) 1396

[12] P.Mantica et al., "Progress in understanding heat transport at JET", Fusion energy 2004, 20th IAEA, Vilamoura 2004 EX/P6-18 Publisher:IAEA (2004) http://wwwpub.iaea.org/MTCD/Meetings/PDFplus/fusion-20-preprints/index.htm

[13] F.Ryter et al., Phys. Rev. Letters, 95 (2005) 085001

[14] A.G. Peeters et al., Phys. Plasmas 12 (2005) 022505

[15] A.Jacchia et al., Nucl. Fusion 45 (2005) 40

[16] A.Jacchia et al., Phys. Fluids B 3 ((1991) 3033

[17] G. Pereverzev, P.N.Yushmanov, Max-Planck-IPP Report, IPP 5/98 (2002)

[18] K.Kirov et al., Plasma Phys. Control. Fusion 44 (2002) 2584

[19] X.Garbet et al., Plasma Phys. Control. Fusion 46 (2004) 1351

[20] X.Garbet et al., Plasma Phys. Control. Fusion 46 (2004) B557

[21] J.Weiland et al., Plasma Phys. Control. Fusion 47 (2005) 441

[22] S. P. Hirshman, R. J. Hawryluk, B. Birge, Nuclear Fusion 17 (1977) 611

[23] J.Weiland and H.Nordman, Phys. Fluids B 5 (1993) 1669 